\title{
Ccreative
commons
}

ISSN 1855-3966 (printed edn.), ISSN 1855-3974 (electronic edn.)

\author{
ARS MATHEMATICA CONTEMPORANEA 22 (2022) \#P2.04 \\ https://doi.org/10.26493/1855-3974.2522.eb3 \\ (Also available at http://amc-journal.eu)
}

\section{Paired domination stability in graphs}

\author{
Aleksandra Gorzkowska \\ AGH University, Department of Discrete Mathematics, \\ al. Mickiewicza 30, 30-059 Krakow, Poland \\ Michael A. Henning \\ Department of Mathematics and Applied Mathematics, University of Johannesburg, \\ Auckland Park, 2006 South Africa \\ Monika Pilśniak \\ AGH University, Department of Discrete Mathematics, \\ al. Mickiewicza 30, 30-059 Krakow, Poland \\ Elżbieta Tumidajewicz * \\ AGH University, Department of Discrete Mathematics, \\ al. Mickiewicza 30, 30-059 Krakow, Poland, and \\ Department of Mathematics and Applied Mathematics, University of Johannesburg, \\ Auckland Park, 2006 South Africa
}

Received 29 December 2020, accepted 16 July 2021, published online 27 May 2022

\begin{abstract}
A set $S$ of vertices in a graph $G$ is a paired dominating set if every vertex of $G$ is adjacent to a vertex in $S$ and the subgraph induced by $S$ contains a perfect matching (not necessarily as an induced subgraph). The paired domination number, $\gamma_{\mathrm{pr}}(G)$, of $G$ is the minimum cardinality of a paired dominating set of $G$. A set of vertices whose removal from $G$ produces a graph without isolated vertices is called a non-isolating set. The minimum cardinality of a non-isolating set of vertices whose removal decreases the paired domination number is the $\gamma_{\mathrm{pr}}^{-}$-stability of $G$, denoted $\operatorname{st}_{\gamma_{\mathrm{pr}}}^{-}(G)$. The paired domination stability of $G$ is the minimum cardinality of a non-isolating set of vertices in $G$ whose removal changes the paired domination number. We establish properties of paired domination stability in graphs. We prove that if $G$ is a connected graph with $\gamma_{\mathrm{pr}}(G) \geq 4$, then $\operatorname{st}_{\gamma_{\mathrm{pr}}}(G) \leq 2 \Delta(G)$ where $\Delta(G)$ is the maximum degree in $G$, and we characterize the infinite family of trees that achieve equality in this upper bound.
\end{abstract}

Keywords: Paired domination, paired domination stability.

\footnotetext{
*Corresponding author.
} 


\section{Introduction}

In 1983 Bauer, Harary, Nieminen and Suffel [3] introduced and studied the concept of domination stability in graphs. Stability for other domination type parameters has been studied in the literature. For example, total domination stability, 2-rainbow domination stability, exponential domination stability, Roman domination stability are studied in [1, 2, $12,15,16]$, among other papers. In this paper we study the paired version of domination stability.

Let $G=(V, E)$ be a graph with vertex set $V=V(G)$ and edge set $E=E(G)$. Two vertices $u$ and $v$ are neighbors if they are adjacent, that is, if $u v \in E$. A dominating set of $G$ is a set $D$ of vertices such that every vertex in $V(G) \backslash D$ has a neighbor in $D$. The minimum cardinality of a dominating set is the domination number, $\gamma(G)$, of $G$. Domination is well studied in the literature. A recent book on domination in graphs can be found in [10]. A small sample of papers on domination critical graphs can be found in [3, 4, 5, 6, 9, 17, 18]. Adopting the notation coined by Bauer et al. [3], the $\gamma^{-}$-stability $\left(\gamma^{+}\right.$-stability, resp. $)$of $G$, denoted by $\gamma^{-}(G)\left(\gamma^{+}(G)\right.$, resp.), is the minimum number of vertices whose removal decreases (increases, resp.) the domination number. The minimum number of vertices whose removal decreases or increases the domination number is the domination stability, $\operatorname{st}_{\gamma}(G)$, of $G$, and so $\operatorname{st}_{\gamma}(G)=\min \left\{\gamma^{-}(G), \gamma^{+}(G)\right\}$.

We refer to a graph without isolated vertices as an isolate-free graph. Unless otherwise stated, let $G$ be an isolate-free graph. A total dominating set, abbreviated TD-set, of $G$ is a set $D$ of vertices of $G$ such that every vertex, including vertices in the set $D$, has a neighbor in $D$. The minimum cardinality of a TD-set of $G$ is the total domination number, $\gamma_{t}(G)$, of $G$. We call a TD-set of $G$ of cardinality $\gamma_{t}(G)$ a $\gamma_{t}$-set of $G$. A vertex $v$ is totally dominated by a set $D$ in $G$ if the vertex $v$ has a neighbor in $D$. We refer the reader to the book [14] for fundamental concepts on total domination in graphs. Total domination critical graphs are studied, for example, in [7, 13]. The total version of domination stability was first studied by Henning and Krzywkowski [12].

A paired dominating set, abbreviated PD-set, of an isolate-free graph $G$ is a dominating set $S$ of $G$ with the additional property that the subgraph $G[S]$ induced by $S$ contains a perfect matching $M$ (not necessarily induced). With respect to the matching $M$, two vertices joined by an edge of $M$ are paired and are called partners in $S$. The paired domination number, $\gamma_{\mathrm{pr}}(G)$, of $G$ is the minimum cardinality of a PD-set of $G$. We call a PD-set of $G$ of cardinality $\gamma_{\mathrm{pr}}(G)$ a $\gamma_{\mathrm{pr}}$-set of $G$. We note that the paired domination number $\gamma_{\mathrm{pr}}(G)$ is an even integer. For a recent survey on paired domination in graphs, we refer the reader to the book chapter [8].

Every PD-set is a TD-set, implying that $\gamma_{t}(G) \leq \gamma_{\mathrm{pr}}(G)$. A non-isolating set of vertices in $G$ is a set $S \subseteq V$ such that the graph $G-S$ is isolate-free, where $G-S$ is the graph obtained from $G$ by removing $S$ and all edges incident with vertices in $S$. Let $\operatorname{NI}(G)$ denote the set of all non-isolating sets of vertices of $G$.

Adopting the standard notation for domination stability given in [3, 12], the $\gamma_{\mathrm{pr}}^{-}$-stability

E-mail addresses: agorzkow@agh.edu.pl (Aleksandra Gorzkowska), mahenning@uj.ac.za (Michael A. Henning), pilsniak@agh.edu.pl (Monika Pilśniak), etumid@agh.edu.pl (Elżbieta Tumidajewicz) 
(resp., $\gamma_{\mathrm{pr}}^{+}$-stability) of $G$, denoted by st $\bar{\gamma}_{\mathrm{pr}}(G)$ (resp., $\mathrm{st}_{\gamma_{\mathrm{pr}}}^{+}(G)$ ) is the minimum cardinality of a set in $\mathrm{NI}(G)$ whose removal decreases (increases, resp.) the paired domination number. Thus,

$$
\text { st }_{\gamma_{\mathrm{pr}}}^{-}(G)=\min _{S \in \mathrm{NI}(G)}\left\{|S|: \gamma_{\mathrm{pr}}(G-S)<\gamma_{\mathrm{pr}}(G)\right\}
$$

and

$$
\operatorname{st}_{\gamma_{\mathrm{pr}}}^{+}(G)=\min _{S \in \mathrm{NI}(G)}\left\{|S|: \gamma_{\mathrm{pr}}(G-S)>\gamma_{\mathrm{pr}}(G)\right\} .
$$

If there is no set in $\mathrm{NI}(G)$ whose removal increases the paired domination number, then we define $\mathrm{st}_{\gamma_{\mathrm{pr}}}^{+}(G)=\infty$. For example, $\mathrm{st}_{\gamma_{\mathrm{pr}}}^{-}\left(P_{5}\right)=1$ while $\mathrm{st}_{\gamma_{\mathrm{pr}}}^{+}\left(P_{5}\right)=\infty$. The paired domination stability, $\operatorname{st}_{\gamma_{\mathrm{pr}}}(G)$, of $G$ is the minimum cardinality of a set in $\mathrm{NI}(G)$ whose removal increases or decreases the paired domination number. Thus,

$$
\operatorname{st}_{\gamma_{\mathrm{pr}}}(G)=\min _{S \in \mathrm{NI}(G)}\left\{|S|: \gamma_{\mathrm{pr}}(G-S) \neq \gamma_{\mathrm{pr}}(G)\right\}=\min \left\{\mathrm{st}_{\gamma_{\mathrm{pr}}}^{-}(G), \mathrm{st}_{\gamma_{\mathrm{pr}}}^{+}(G)\right\}
$$

Let $G$ be a graph and let $S \in \mathrm{NI}(G)$. If $\gamma_{\mathrm{pr}}(G-S)<\gamma_{\mathrm{pr}}(G)$ and $|S|=\operatorname{st}_{\gamma_{\mathrm{pr}}}^{-}(G)$, then we call $S$ a st $\gamma_{\mathrm{pr}}$-set of $G$. If $\gamma_{\mathrm{pr}}(G-S)>\gamma_{\mathrm{pr}}(G)$ and $|S|=\operatorname{st}_{\gamma_{\mathrm{pr}}}^{+}(G)$, then we call $S$ a st $\gamma_{\mathrm{pr}}$-set of $G$. If $\gamma_{\mathrm{pr}}(G-S) \neq \gamma_{\mathrm{pr}}(G)$ and $|S|=\operatorname{st}_{\gamma_{\mathrm{pr}}}(G)$, then we call $S$ a st $\gamma_{\mathrm{pr}}$-set of $G$.

Defining the null graph $K_{0}$, which has no vertices, as a graph, we have the following results due to Bauer et al. [3] and Rad et al. [15] for the $\gamma^{-}$-stability of a graph.

Theorem 1.1 ([3, 15]). If $G$ is an isolate-free graph of order $n$, then the following holds.

(a) $\operatorname{st}_{\gamma}(G) \leq \delta(G)+1$.

(b) If $G ¥ K_{n}$, then $\operatorname{st}_{\gamma}(G) \leq n-1$.

Considering the null graph, the paired domination stability of a non-trivial graph is always defined. If $G$ is a graph of order $n$ and $\gamma_{\mathrm{pr}}(G)=2$, then $\operatorname{st}_{\gamma_{\mathrm{pr}}}^{-}(G)=n$ since removing all vertices from the graph $G$ produces the null graph with paired domination number zero.

For notation and graph theory terminology we generally follow [14]. In particular, for $r, s \geq 1$, a double star $S(r, s)$ is the tree with exactly two vertices that are not leaves, one of which has $r$ leaf-neighbors and the other $s$ leaf-neighbors. A rooted tree is a tree $T$ in which we specify one vertex $r$ called the root. For each vertex $v$ of $T$ different from $r$, its parent is the neighbor of $v$ on the unique $(r, v)$-path, while every other neighbor of $v$ is a child of $v$ in $T$. If $w$ is a vertex of $T$ different from $v$ and the (unique) $(r, w)$-path contains $v$, then $w$ is a descendant of $v$ in $T$. We note that every child of $v$ is a descendant of $v$. The diameter $\operatorname{diam}(G)$ of $G$ is the maximum distance among all pairs of vertices of $G$. A diametral path in $G$ is a shortest path between two vertices in $G$ of length equal to $\operatorname{diam}(G)$. For an integer $k \geq 1,[k]=\{1, \ldots, k\}$.

\section{Main results}

Our first aim is to show that the paired domination stability of a graph can be very different from its total domination stability studied in [12].

Theorem 2.1. For $k \geq 1$ an arbitrary integer, the following holds. 
(a) There exist connected graphs $G$ such that $\mathrm{st}_{\gamma_{\mathrm{pr}}}^{-}(G)-\mathrm{st}_{\gamma_{t}}^{-}(G)=k$.

(b) There exist connected graphs $H$ such that $\mathrm{st}_{\gamma_{t}}^{-}(H)-\mathrm{st}_{\gamma_{\mathrm{pr}}}^{-}(H)=k$.

Our second aim is to establish properties of paired domination stability in graphs. Thereafter, we establish upper bounds on the paired domination stability and the $\gamma_{\mathrm{pr}^{-}}^{-}$ stability of a graph. For this purpose, we shall need the following family of trees defined by Henning and Krzywkowski [12]. For integers $k \geq 2$ and $\Delta \geq 2$, the authors in [12] define $T_{k, \Delta}$ as the "graph obtained from the disjoint union of $k$ double stars $S(\Delta-1, \Delta-1)$ by adding $k-1$ edges between the leaves of these double stars so that the resulting graph is a tree with maximum degree $\Delta$." Let $\mathcal{F}_{k, \Delta}$ be the family of all such trees $T_{k, \Delta}$, and let

$$
\mathcal{F}_{\Delta}=\bigcup_{k \geq 2} \mathcal{F}_{k, \Delta} .
$$

The following result establishes an upper bound on the $\gamma_{\mathrm{pr}}^{-}$-stability of a tree, and characterizes the trees with maximum possible $\gamma_{\mathrm{pr}}^{-}$-stability.

Theorem 2.2. If $T$ is a tree with maximum degree $\Delta$ satisfying $\gamma_{\mathrm{pr}}(T) \geq 4$, then the following hold.

(a) $\operatorname{st}_{\gamma_{\mathrm{pr}}}^{-}(T) \leq 2 \Delta$, with equality if and only if $T \in \mathcal{F}_{\Delta}$.

(b) $\operatorname{st}_{\gamma_{\mathrm{pr}}}(T) \leq 2 \Delta-1$, and this bound is sharp for all $\Delta \geq 2$.

For general graphs, we establish the following upper bound on the $\gamma_{\mathrm{pr}}^{-}$-stability in terms of the maximum degree of the graph.

Theorem 2.3. If $G$ is a connected graph with $\gamma_{\mathrm{pr}}(G) \geq 4$, then $\mathrm{st}_{\gamma_{\mathrm{pr}}}^{-}(G) \leq 2 \Delta(G)$, and this bound is sharp.

As an immediate consequence of Theorem 2.3, we have the following upper bound on the paired domination stability of a graph.

Corollary 2.4. If $G$ is a connected graph with $\gamma_{\mathrm{pr}}(G) \geq 4$, then $\operatorname{st}_{\gamma_{\mathrm{pr}}}(G) \leq 2 \Delta(G)$.

\section{Paired stability versus domination and total stability}

In this section, we show that paired domination stability and the domination stability of a graph can be very different. By Theorem 1.1, for every nontrivial graph $G$, we have $\operatorname{st}_{\gamma}(G) \leq \delta(G)+1$. In particular, $\operatorname{st}_{\gamma}(T) \leq 2$ for every nontrivial tree $T$. This is in contrast to the paired domination stability, where for any given $\Delta \geq 2$, we show that there exist a family of trees $T$ with maximum degree $\Delta$ satisfying st $\gamma_{\mathrm{pr}}(T)=2 \Delta-1$.

For $\Delta=2$, the authors in [12] define $\mathcal{H}_{\Delta}$ as the family of all paths of order at least 7 and congruent to 3 modulo 4 , that is, $\mathcal{H}_{\Delta}=\left\{P_{n} \mid n \equiv 3(\bmod 4)\right.$ and $\left.n \geq 7\right\}$. For integers $\Delta \geq 3$ and $\Delta \geq k \geq 2$, they define $H_{k, \Delta}$ as the graph "obtained from the disjoint union of $k$ double stars $S(\Delta-1, \Delta-1)$ by selecting one leaf from each double star and identifying these $k$ leaves into one new vertex" and they define the family

$$
\mathcal{H}_{\Delta}=\bigcup_{k \geq 2} H_{k, \Delta} .
$$

We determine next the paired domination stability of a tree in the family $\mathcal{H}_{\Delta}$. 
Proposition 3.1. For $\Delta \geq 3$, if $T \in \mathcal{H}_{\Delta}$, then $\operatorname{st}_{\gamma_{\mathrm{pr}}}(T)=2 \Delta-1$.

Proof. For integers $\Delta \geq k \geq 2$ where $\Delta \geq 3$, consider a tree $T \in \mathcal{H}_{k, \Delta}$. By definition of the family $\mathcal{H}_{k, \Delta}$, the tree $T$ is constructed from the disjoint union of $k$ double stars $S_{1}, \ldots, S_{k}$, each isomorphic to $S(\Delta-1, \Delta-1)$, by selecting one leaf from each double star and identifying these $k$ chosen leaves into one new vertex, which we call $v_{c}$. Let $x_{i}$ and $y_{i}$ be the two central vertices of the double star $S_{i}$ for $i \in[k]$, where $x_{i}$ is adjacent to $v_{c}$ in $T$. Let $D=\cup_{i=1}^{k}\left\{x_{i}, y_{i}\right\}$. Since $\Delta \geq 3$, every vertex in $D$ is a support vertex of $T$, implying that every PD-set in $T$ contains the set $D$ and therefore $\gamma_{\mathrm{pr}}(T) \geq|D|=2 k$. Since the set $D$ is a PD-set of $T$ (with the vertices $x_{i}$ and $y_{i}$ paired for all $i \in[k]$ ), we have $\gamma_{\mathrm{pr}}(T) \leq|D|=2 k$. Consequently, $\gamma_{\mathrm{pr}}(T)=2 k$ and $D$ is the unique $\gamma_{\mathrm{pr}}$-set of $T$.

Let $S$ be a st $\gamma_{\mathrm{pr}}$-set of $T$. Thus, $S$ is a set in $\mathrm{NI}(T)$ with $|S|=\operatorname{st}_{\gamma_{\mathrm{pr}}}(T)$ satisfying $\gamma_{\mathrm{pr}}(T-S) \neq \gamma_{\mathrm{pr}}(T)=2 k$. We show that $|S| \geq 2 \Delta-1$. Suppose, to the contrary, that $|S| \leq 2 \Delta-2$. If the set $S$ contains both $x_{i}$ and $y_{i}$ for some $i \in[k]$, then since $S$ is a nonisolating set of $T$ every leaf neighbor of $x_{i}$ and $y_{i}$ is also in $S$, implying that $|S| \geq 2 \Delta-1$, a contradiction. Hence, the set $S$ contains at most one of $x_{i}$ and $y_{i}$ for every $i \in[k]$. Let $D^{*}$ be a $\gamma_{\mathrm{pr}}$-set of $T-S$, and so $\left|D^{*}\right| \neq 2 k$.

Suppose that $v_{c} \in S$. In this case, if $|S|=1$, then the paired domination numbers of $T$ and $T-S$ are the same, a contradiction. Hence, $|S| \geq 2$. If neither $x_{i}$ nor $y_{i}$ belong to $S$ for some $i \in[k]$, then by the minimality of the non-isolating set $S$, no vertex of $T_{i}$ different from $v_{c}$ belongs to $S$, and so $\left|D^{*} \cap V\left(T_{i}\right)\right|=2$. If $S$ contains $y_{i}$ but not $x_{i}$ for some $i \in[k]$, then every leaf neighbor of $y_{i}$ is in $S$ and by the minimality of the set $S$, no leaf neighbor of $x_{i}$ belongs to $S$, and so $\left|D^{*} \cap V\left(T_{i}\right)\right|=2$. Analogously, if $S$ contains $x_{i}$ but not $y_{i}$ for some $i \in[k]$, then $\left|D^{*} \cap V\left(T_{i}\right)\right|=2$. This is true for all $i \in[k]$, implying that $\left|D^{*}\right|=\sum_{i=1}^{k}\left|D^{*} \cap V\left(T_{i}\right)\right|=2 k$, a contradiction. Hence, $v_{c} \notin S$.

As observed earlier, the set $S$ contains at most one of $x_{i}$ and $y_{i}$ for every $i \in[k]$. If $y_{i} \in S$ and $y_{j} \in S$ for some $i, j \in[k]$ where $i \neq j$, then $|S| \geq 2 \Delta$, a contradiction. If $y_{i} \in S$ and $x_{j} \in S$ for some $i, j \in[k]$ where $i \neq j$, then $|S| \geq 2 \Delta-1$, a contradiction. If $x_{i} \in S$ and $x_{j} \in S$ for some $i, j \in[k]$ where $i \neq j$, then $|S| \geq 2 \Delta-2$. In this case, by the minimality of $S$ we have $S=\left(N\left[x_{i}\right] \cup N\left[x_{j}\right]\right) \backslash\left\{v_{c}, y_{i}, y_{j}\right\}$ and $|S|=2 \Delta-2$. But then $T-S$ consists of three components, namely two stars isomorphic to $K_{1, \Delta-1}$ and one component belonging to the family $T \in \mathcal{H}_{k-2, \Delta}$ with paired domination number $2(k-2)$. Thus, $\gamma_{\mathrm{pr}}(T-S)=2+2+2(k-2)=2 k$, a contradiction. Therefore, st $_{\gamma_{\mathrm{pr}}}(T)=|S| \geq$ $2 \Delta-1$, as claimed.

Conversely, if we take $S=N\left(x_{1}\right) \cup N\left(y_{1}\right) \backslash\left\{v_{c}\right\}$, then $S \in \mathrm{NI}(T)$ and $T-S \in$ $\mathcal{H}_{k-1, \Delta}$. Thus, $\gamma_{\mathrm{pr}}(T-S)=2(k-1)<\gamma_{\mathrm{pr}}(T)$, and so st $\gamma_{\mathrm{pr}}(T) \leq \operatorname{st}_{\gamma_{\mathrm{pr}}}^{-}(T) \leq|S|=$ $2 \Delta-1$. Consequently, st $_{\gamma_{\mathrm{pr}}}(T)=\mathrm{st}_{\gamma_{\mathrm{pr}}}^{-}(T)=2 \Delta-1$.

As observed earlier, $\operatorname{st}_{\gamma}(T) \leq 2$ for every nontrivial tree $T$. By Proposition 3.1, paired domination stability therefore differs significantly from domination stability. We show next that the paired domination stability and the total domination stability of a graph can also be very different.

Proposition 3.2. For $k \geq 1$ an integer, there exist trees $T$ such that $\mathrm{st}_{\gamma_{\mathrm{pr}}}^{-}(T)-\mathrm{st}_{\gamma_{t}}^{-}(T)=k$.

Proof. Let $k \geq 1$ be a given integer, and let $T=T_{k}$ be obtained from a path $P_{5}$ given by $v_{1} v_{2} v_{3} v_{4} v_{5}$ by attaching $k$ leaf neighbors to each of $v_{1}, v_{2}$ and $v_{3}$ (see Figure 1 ). We 
note that $\left\{v_{1}, v_{2}, v_{3}, v_{4}\right\}$ is the unique $\gamma_{t}$-set of $T$ and the unique $\gamma_{\mathrm{pr}}$-set of $T$. In particular, $\gamma_{t}(T)=\gamma_{\mathrm{pr}}(T)=4$. If $S=\left\{v_{5}\right\}$, then the set $S$ is a non-isolating set of $T$ and $\gamma_{t}(T-S)=\left|\left\{v_{1}, v_{2}, v_{3}\right\}\right|=3<\gamma_{t}(T)$, implying that $\mathrm{st}_{\gamma_{t}}^{-}(T)=1$.

We show next that st $_{\gamma_{\mathrm{pr}}}^{-}(T)=k+1$. Let $S$ be a non-isolating set of $T$ such that $\gamma_{\mathrm{pr}}(T-S)<\gamma_{\mathrm{pr}}(T)$. We show that $|S| \geq k+1$. Suppose, to the contrary, that $|S| \leq k$. Let $D$ be a $\gamma_{\mathrm{pr}}$-set of $T-S$, and so $|D|=\gamma_{\mathrm{pr}}(T-S)=2$. Let $L_{i}$ denote the set of leaf neighbors of $v_{i}$ for $i \in$ [4]. If $v_{i} \in S$ for some $i \in$ [3], then $S$ contains all $k$ leaf neighbors of $v_{i}$, and so $|S| \geq k+1$, a contradiction. Hence, $S \cap\left\{v_{1}, v_{2}, v_{3}\right\}=\emptyset$. If $\left\{v_{1}, v_{3}\right\} \subset D$, then $|D| \geq 4$, a contradiction. If $v_{1} \notin D$, then $L_{1} \subseteq S$, implying that $S=L_{1}$ and $|S|=k$. However in this case, $\left\{v_{2}, v_{3}, v_{4}\right\} \subset D$. If $v_{3} \notin D$, then $L_{3} \subseteq S$, implying that $S=L_{3}$ and $|S|=k$. However in this case, $\left\{v_{1}, v_{2}, v_{4}\right\} \subset D$. In both cases, $|D| \geq 4$, a contradiction. Therefore, $|S| \geq k+1$, implying that st ${ }_{\gamma_{\mathrm{pr}}}^{-}(T) \geq k+1$. Conversely, if $S=L_{1} \cup L_{4}$, then $S$ is a non-isolating set of $T$ such that $\gamma_{\mathrm{pr}}(T-S)=\left|\left\{v_{2}, v_{3}\right\}\right|<$ $\gamma_{\mathrm{pr}}(T)$, implying that $\mathrm{st}_{\gamma_{\mathrm{pr}}}^{-}(T) \leq|S|=k+1$. Consequently, $\mathrm{st}_{\gamma_{\mathrm{pr}}}^{-}(T)=k+1$. Thus, $\mathrm{st}_{\gamma_{\mathrm{pr}}}^{-}(T)-\mathrm{st}_{\gamma_{t}}^{-}(T)=k$.

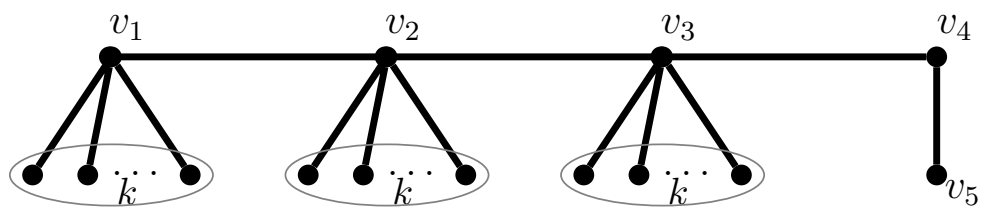

Figure 1: A tree from the family $T_{k}$ in the proof of Proposition 3.2.

Proposition 3.3. For $k \geq 1$ an integer, there exist trees $T$ such that $\mathrm{st}_{\gamma_{t}}^{-}(T)-\mathrm{st}_{\gamma_{\mathrm{pr}}}^{-}(T)=k$.

Proof. Let $k \geq 1$ be a given integer, and let $\ell \geq 2 k+1$ be an integer. For $i \in[k]$, let $Q_{i}$ be obtained from a path $v_{i_{1}} v_{i_{2}} v_{i_{3}} v_{i_{4}} v_{i_{5}}$ by attaching $\ell$ leaf neighbors to each of $v_{i_{3}}, v_{i_{4}}$ and $v_{i_{5}}$, and let $L_{i_{3}}, L_{i_{4}}$ and $L_{i_{5}}$ be the resulting sets of leaf neighbors of $v_{i_{3}}, v_{i_{4}}$ and $v_{i_{5}}$, respectively. Let $Q$ be obtained from a path $v_{1} v_{2} v_{3}$ by attaching $\ell$ leaf neighbors to each of $v_{1}$ and $v_{2}$, and attaching $k$ leaf neighbors to $v_{3}$. Let $L_{i}$ be the resulting set of leaf neighbors of $v_{i}$ for $i \in[3]$. Let $T$ be obtained from the disjoint union of the paths $Q, Q_{1}, \ldots, Q_{k}$ by adding the $k$ edges $v_{3} v_{i_{1}}$ for $i \in[k]$. Let $A$ be the set of support vertices of $T$, and so $|A|=3(k+1)$.

Every TD-set of $T$ contains all its support vertices, implying that $\gamma_{t}(T) \geq|A|$. Since the set $A$ is a TD-set of $T$, we have $\gamma_{t}(T) \leq|A|$. Consequently, $\gamma_{t}(T)=|A|=3(k+1)$. Every PD-set of $T$ contains the set $A$ and at least one additional vertex from each path $Q_{i}$ that is a neighbor of $v_{i_{3}}$ or $v_{i_{5}}$ for $i \in[k]$, and at least one additional vertex that is a neighbor of $v_{1}$ or $v_{3}$ since the vertices of every PD-set are paired, implying that $\gamma_{\mathrm{pr}}(T)=$ $|A|+k+1=4(k+1)$.

Let $S$ be a non-isolating set of $T$ such that $\gamma_{\mathrm{pr}}(T-S)<\gamma_{\mathrm{pr}}(T)$. If $|S|<k$, then every support vertex of $T$ remains a support vertex of $T-S$, implying that $\gamma_{\mathrm{pr}}(T-S) \geq \gamma_{\mathrm{pr}}(T)$, a contradiction. Hence, $|S| \geq k$. Conversely, if $S^{*}=L_{3}$, then the set $A \backslash\left\{v_{3}\right\}$ of all support vertices of $T-S^{*}$, together with the vertices $v_{i_{2}}$ for $i \in[k]$, form a PD-set of $T-S^{*}$, implying that $\gamma_{\mathrm{pr}}\left(T-S^{*}\right) \leq 4 k+2<4 k+4=\gamma_{\mathrm{pr}}(T)$. Hence, $\mathrm{st}_{\gamma_{\mathrm{pr}}}^{-}(T) \leq\left|S^{*}\right|=k$. Consequently, $\mathrm{st}_{\gamma_{\mathrm{pr}}}^{-}(T)=k$. 
We show next that $\operatorname{st}_{\gamma_{t}}^{-}(T)=2 k$. Let $A^{\prime}=A \backslash\left\{v_{3}\right\}$, and so $\left|A^{\prime}\right|=|A|-1=$ $3 k+2$. Let $S$ be a non-isolating set of $T$ such that $\gamma_{t}(T-S)<\gamma_{t}(T)$. We show that $|S| \geq 2 k$. Suppose, to the contrary, that $|S| \leq 2 k-1$. Let $D$ be a $\gamma_{t}$-set of $T-S$, and so $|D|=\gamma_{t}(T-S) \leq 3 k+2$. Since $|S|<2 k<\ell$ and each vertex in $A^{\prime}$ has $\ell$ leaf neighbors in $T$, we note that every vertex of $A^{\prime}$ is a support vertex of $T-S$, implying that $A^{\prime} \subseteq D$, and so $3 k+2 \geq|D| \geq\left|A^{\prime}\right|=3 k+2$, implying that $D=A^{\prime}$. In particular, $v_{3} \notin D$, implying that all $k$ leaf neighbors of $v_{3}$ belong to $S$; that is, $L_{3} \subseteq S$. If $v_{i_{1}} \notin S$ for some $i \in[k]$, then in order to totally dominate the vertex $v_{i_{1}}$, the vertex $v_{i_{2}} \in D$, contradicting our earlier observation that $D=A^{\prime}$. Hence, $v_{i_{1}} \in S$ for all $i \in[k]$, and so $|S| \geq\left|L_{3}\right|+k=2 k$, a contradiction. Therefore, our original supposition that $|S| \leq 2 k-1$ is incorrect, implying that $|S| \geq 2 k$ and $\operatorname{st}_{\gamma_{\mathrm{pr}}}^{-}(T) \geq 2 k$. Conversely, if $S^{*}$ consists of all $2 k$ neighbors of $v_{3}$ different from $v_{2}$ in $T$, then $S^{*}$ is a non-isolating set of $T$ such that $\gamma_{t}\left(T-S^{*}\right)=\left|A^{\prime}\right|<\gamma_{t}(T)$, implying that $\operatorname{st}_{\gamma_{t}}^{-}(T) \leq\left|S^{*}\right|=2 k$. Consequently, st $_{\gamma_{t}}^{-}(T)=2 k$. Thus, st $_{\gamma_{t}}^{-}(T)-$ st $_{\gamma_{\mathrm{pr}}}^{-}(T)=k$.

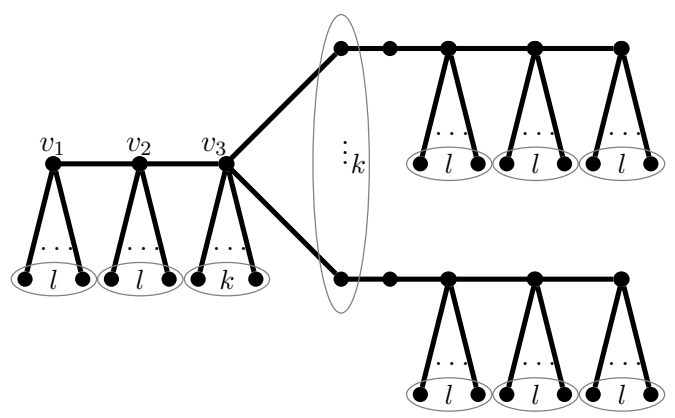

Figure 2: A tree from the family $T$ in the proof of Proposition 3.3.

Theorem 2.1 follows from Propositions 3.2 and 3.3. As further examples, we remark that if $P$ is the Petersen graph, then $\gamma_{t}(P)=4$ and $\gamma_{\mathrm{pr}}(P)=6$. Further, if $v$ is an arbitrary vertex of $P$, then $\gamma_{t}(P-v)=4$, and so $\operatorname{st}_{\gamma_{t}}^{-}(P) \geq 2$. Moreover, if $S$ consists of two non-adjacent vertices of $P$, then $\gamma_{t}(P-S)=3$, and so st $\gamma_{t}(P) \leq 2$. Consequently, $\operatorname{st}_{\gamma_{t}}^{-}(P)=2$. However if $v$ is an arbitrary vertex of $P$, then $\gamma_{\mathrm{pr}}(P-v)=4$, implying that st $_{\gamma_{\mathrm{pr}}}^{-}(P)=1$. Moreover, let $G_{k}$ be a graph obtained from the Petersen graph by replacing every vertex by a copy of a complete graph $K_{k}$ for some $k \geq 1$, and adding all edges between two resulting complete graphs that correspond to two vertices of $G_{k}$ (see Fig. 3). The resulting graph $G_{k}$ is a $(4 k-1)$-regular, $3 k$-connected graph that satisfies $\gamma_{t}\left(G_{k}\right)=4$ and $\operatorname{st}_{\gamma_{t}}^{-}\left(G_{k}\right)=2 k$, and $\gamma_{\mathrm{pr}}\left(G_{k}\right)=6$ and $\operatorname{st}_{\gamma_{\mathrm{pr}}}^{-}\left(G_{k}\right)=k$. This yields the following result.

Proposition 3.4. For $k \geq 1$ an integer, there exists $(4 k-1)$-regular, $3 k$-connected graphs G such that $\mathrm{st}_{\gamma_{t}}^{-}(G)-\mathrm{st}_{\gamma_{\mathrm{pr}}}^{-}(G)=k$.

\section{Properties of paired domination stability}

In this section, we present properties of paired domination stability in graphs. We begin with the following property of paired domination in graphs. 


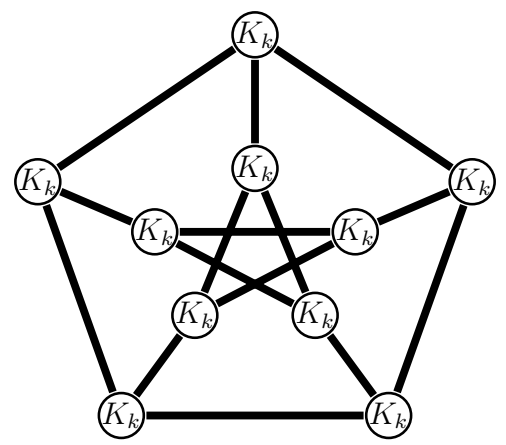

Figure 3: A graph $G_{k}$ obtained from the Petersen graph by replacing every vertex by $K_{k}$.

Proposition 4.1. Every connected isolate-free graph $G$ contains a spanning tree $T$ such that $\gamma_{\mathrm{pr}}(T)=\gamma_{\mathrm{pr}}(G)$.

Proof. Since adding edges to a graph cannot increases its paired domination number, if $T$ is an isolate-free spanning subgraph of a graph $G$, then $\gamma_{\mathrm{pr}}(G) \leq \gamma_{\mathrm{pr}}(T)$. Let $D$ be a $\gamma_{\mathrm{pr}}$-set of $G$, and so $D$ is a PD-set of $G$ and $|D|=\gamma_{\mathrm{pr}}(G)$. Let $M$ be a perfect matching in the subgraph $G[D]$ induced by $D$. Let $T^{\prime}$ be a spanning subgraph of $G$ that consists of the edges in $M$ and for each vertex $v$ outside $D$, an edge of $G$ that joins $v$ to exactly one vertex of the dominating set $D$. If the resulting spanning subgraph $T^{\prime}$ is a tree, then we let $T=T^{\prime}$. Otherwise, if the resulting spanning subgraph $T^{\prime}$ is a forest with $\ell \geq 2$ components, then we add $\ell-1$ edges from the edge set of the graph $G$ between these components, avoiding cycles, to construct a tree, which we call $T$. Since $D$ is a PD-set in the resulting tree $T$, we note that $\gamma_{\mathrm{pr}}(T) \leq|D|=\gamma_{\mathrm{pr}}(G)$. Since $T$ is an isolate-free spanning subgraph of $G$, we have $\gamma_{\mathrm{pr}}(T) \geq \gamma_{\mathrm{pr}}(G)$. Consequently, $T$ is a spanning tree of $G$ satisfying $\gamma_{\mathrm{pr}}(T)=\gamma_{\mathrm{pr}}(G)$.

By our earlier convention, if $G$ is a graph of order $n$ and $\gamma_{\mathrm{pr}}(G)=2$, then $\operatorname{st}_{\gamma_{\mathrm{pr}}}^{-}(G)=n$ since removing all vertices from the graph $G$ produces the null graph with paired domination number zero. We are therefore only interested in the $\gamma_{\mathrm{pr}}^{-}$-stability of graphs with paired domination number at least 4 . If $G$ is a graph with $\gamma_{\mathrm{pr}}(G) \geq 4$ where $x$ and $y$ are adjacent vertices in $G$, then $D=V(G) \backslash\{x, y\}$ belongs to the set $\mathrm{NI}(G)$ and $\gamma_{\mathrm{pr}}(G-D)=\gamma_{\mathrm{pr}}\left(K_{2}\right)=2<\gamma_{\mathrm{pr}}(G)$. This yields the following result.

Observation 4.2. Every isolate-free graph $G$ of order $n$ with $\gamma_{\mathrm{pr}}(G) \geq 4$ satisfies st $_{\gamma_{\mathrm{pr}}}^{-}(G) \leq n-2$.

Proposition 4.3. If $T$ is a spanning tree of a connected graph $G$ such that $\gamma_{\mathrm{pr}}(T)=\gamma_{\mathrm{pr}}(G)$, then $\mathrm{st}_{\gamma_{\mathrm{pr}}}^{-}(T) \geq \mathrm{st}_{\gamma_{\mathrm{pr}}}^{-}(G)$.

Proof. Let $S$ be a st $\bar{\gamma}_{\mathrm{pr}}$-set of $T$. Thus, $S$ is a set in $\mathrm{NI}(T)$ with $|S|=\mathrm{st}_{\gamma_{\mathrm{pr}}}^{-}(T)$ such that $\gamma_{\mathrm{pr}}(T-S)<\gamma_{\mathrm{pr}}(T)$. Since $\gamma_{\mathrm{pr}}(G-S) \leq \gamma_{\mathrm{pr}}(T-S)$ and $\gamma_{\mathrm{pr}}(T) \stackrel{\mathrm{pr}}{=} \gamma_{\mathrm{pr}}(G)$, the set $S$ is a non-isolating set of $G$ such that $\gamma_{\mathrm{pr}}(G-S)<\gamma_{\mathrm{pr}}(G)$. Hence, $\operatorname{st}_{\gamma_{\mathrm{pr}}}^{-}(G) \leq$ $|S|=\operatorname{st}_{\gamma_{\mathrm{pr}}}^{-}(T)$.

The following result shows that to determine the $\gamma_{\mathrm{pr}}^{-}$-stability of a graph $G$, it is not sufficient to only examine spanning trees $T$ of $G$ satisfying $\gamma_{\mathrm{pr}}(T)=\gamma_{\mathrm{pr}}(G)$. 
Proposition 4.4. For $k \geq 1$ an integer, there exist connected graphs $G$ such that $\mathrm{st}_{\gamma_{\mathrm{pr}}}^{-}(T)-\mathrm{st}_{\gamma_{\mathrm{pr}}}^{-}(G)=k$ for every spanning tree $T$ of $G$ with $\gamma_{\mathrm{pr}}(T)=\gamma_{\mathrm{pr}}(G)$.

Proof. For $k \geq 1$, let $F$ be obtained from two vertex disjoint copies of $K_{2, k+1}$ by identifying a vertex of degree $k+1$ from each copy. Let $u$ be the resulting identified vertex of degree $2(k+1)$, and let $w_{1}$ and $w_{2}$ be the two vertices of degree $k+1$ in $F$. Further, let $v_{i}$ be a common neighbor (of degree 2) of $u$ and $w_{i}$ for $i \in[2]$. Let $G$ be obtained from $F$ by adding a leaf neighbor $x_{i}$ to $w_{i}$ for $i \in[2]$. Thus, $\operatorname{diam}(G)=6$ and $x_{1} w_{1} v_{1} u v_{2} w_{2} x_{2}$ is a shortest path in $G$ of length 6 . The graph $G$ satisfies $\gamma_{\mathrm{pr}}(G)=4$. We remark that only connected graphs of $\operatorname{diam}(G) \leq 3$ have $\gamma_{\mathrm{pr}}(G)=2$. Therefore, st $_{\gamma_{\mathrm{pr}}}^{-}(G) \geq 3$. Moreover, the set $S=\left\{w_{1}, x_{1}, x_{2}\right\}$ is a non-isolating set of minimum cardinality satisfying $\gamma_{\mathrm{pr}}(G-S)=2<\gamma_{\mathrm{pr}}(G)$, and so $\operatorname{st}_{\gamma_{\mathrm{pr}}}^{-}(G)=3$. However, the vertex $u$ must have degree 2 in every spanning tree $T$ of $G$ for which $\gamma_{\mathrm{pr}}(T)=\gamma_{\mathrm{pr}}(G)=4$, implying that the vertices $w_{1}$ and $w_{2}$ each have $k+1$ leaf neighbors in $T$. This implies that every non-isolating set of $T$ that decreases the paired domination number contains at least $k+3$ vertices. The set $S=N_{T}\left[w_{1}\right]$ is a non-isolating set of minimum cardinality satisfying $\gamma_{\mathrm{pr}}(T-S)=2<\gamma_{\mathrm{pr}}(T)$, and so st ${ }_{\gamma_{\mathrm{pr}}}^{-}(T) \leq|S|=k+3$. Consequently, $\mathrm{st}_{\gamma_{\mathrm{pr}}}^{-}(T)=k+3$, and so st $\bar{\gamma}_{\mathrm{pr}}(T)-\mathrm{st}_{\gamma_{\mathrm{pr}}}^{-}(G)=k$.

Proposition 4.5. If $S$ is a $\mathrm{st}_{\gamma_{\mathrm{pr}}}^{-}$-set of a connected isolate-free graph $G$ with $\gamma_{\mathrm{pr}}(G) \geq 4$, then $\gamma_{\mathrm{pr}}(G-S)=\gamma_{\mathrm{pr}}(G)-2$.

Proof. Let $S$ be a st ${ }_{\gamma_{\mathrm{pr}}}^{-}$-set of $G$. Suppose, to the contrary, that $\gamma_{\mathrm{pr}}(G-S) \leq \gamma_{\mathrm{pr}}(G)-4$. By the connectivity of $G$, there exists a vertex $u \in S$ that has a neighbor in the set $V(G) \backslash S$. We now consider the set $S^{\prime}=S \backslash\{u\}$. Let $D$ be a $\gamma_{\mathrm{pr}}$-set of $G-S$. If $u$ has a neighbor in $D$, then $D$ is a $\gamma_{\mathrm{pr}}$-set of $G-S^{\prime}$, implying that $\gamma_{\mathrm{pr}}\left(G-S^{\prime}\right) \leq|D|=\gamma_{\mathrm{pr}}(G-S) \leq \gamma_{\mathrm{pr}}(G)-4$, contradicting our choice of the set $S$. Hence, $u$ has no neighbor in $D$. Let $v$ be an arbitrary neighbor of $u$ that belongs to $V(G) \backslash S$. The set $D \cup\{u, v\}$ is a PD-set of $G-S^{\prime}$ with $u$ and $v$ paired, and with the pairings of the vertices of $D$ unchanged from their pairings in $G-S$. Hence, $\gamma_{\mathrm{pr}}\left(G-S^{\prime}\right) \leq|D|+2 \leq \gamma_{\mathrm{pr}}(G)-2$, once again contradicting our choice of the set $S$.

\section{Paths and cycles}

It is well known (see, for example, [11]) that for $n \geq 3$ we have $\gamma_{\mathrm{pr}}\left(C_{n}\right)=\gamma_{\mathrm{pr}}\left(P_{n}\right)=$ $2\left\lceil\frac{n}{4}\right\rceil$. In this section, we determine the paired domination stability of paths and cycles. The proofs require a detailed case analysis, which is straightforward albeit tedious. We therefore omit the proofs in this section. The $\gamma_{\mathrm{pr}}^{-}$-stability of a path $P_{n}$ and a cycle $C_{n}$ on $n$ vertices is given by the following result.

Theorem 5.1. If $G$ is a path $P_{n}$, for $n \geq 2$, or a cycle $C_{n}$, for $n \geq 3$, then

$$
\text { st }_{\gamma_{\mathrm{pr}}}^{-}(G)= \begin{cases}1 & \text { when } n \equiv 1(\bmod 4) \\ 2 & \text { when } n \equiv 2(\bmod 4) \\ 3 & \text { when } n \equiv 3(\bmod 4) \\ 4 & \text { when } n \equiv 0(\bmod 4)\end{cases}
$$

Next we determine the $\gamma_{\mathrm{pr}}^{+}$-stability of a path $P_{n}$. For $n \leq 10$ with $n \neq 8$ and for $n=13$, no non-isolating set of vertices in a path $P_{n}$ exists whose removal increases the 
paired domination number, and hence, by definition, $\operatorname{st}_{\gamma_{\mathrm{pr}}}^{+}\left(P_{n}\right)=\infty$ for such values of $n$. It is therefore only of interest to determine the $\gamma_{\mathrm{pr}}^{+}$-stability of a path $P_{n}$, where $n \geq 8$ and $n \notin\{9,10,13\}$.

Theorem 5.2. For $n \geq 8$ and $n \notin\{9,10,13\}$,

$$
\mathrm{st}_{\gamma_{\mathrm{pr}}}^{+}\left(P_{n}\right)= \begin{cases}1 & \text { when } n(\bmod 4) \in\{0,3\} \\ 2 & \text { when } n(\bmod 4) \in\{1,2\} .\end{cases}
$$

As a consequence of Theorems 5.1 and 5.2, the paired domination stability of a path is determined.

Corollary 5.3. For $n \geq 2$,

$$
\operatorname{st}_{\gamma_{\mathrm{pr}}}\left(P_{n}\right)= \begin{cases}1 & \text { when } n(\bmod 4) \in\{0,1,3\} \text { and } n \notin\{3,4,7\} \\ 2 & \text { when } n \equiv 2(\bmod 4) \\ 3 & \text { when } n \in\{3,7\} \\ 4 & \text { when } n=4 .\end{cases}
$$

We next consider the $\gamma_{\mathrm{pr}}^{+}$-stability of a cycle $C_{n}$. As shown in Theorem 5.1, the $\gamma_{\mathrm{pr}^{-}}^{-}$ stability of a path and a cycle of the same order are equal. This is not always the case for the $\gamma_{\mathrm{pr}}^{+}$-stability of a path and a cycle. For example, $\mathrm{st}_{\gamma_{\mathrm{pr}}}^{+}\left(P_{12}\right)=1$ and $\mathrm{st}_{\gamma_{\mathrm{pr}}}^{+}\left(C_{12}\right)=2$. Analogously as in the case of paths, for small values of the order of a cycle the $\gamma_{\mathrm{pr}}^{+}$-stability is infinite. Namely, for $n \leq 14$ with $n \neq 12$ and $n=17$ we have that $\operatorname{st}_{\gamma_{\mathrm{pr}}}^{+}\left(C_{n}\right)=\infty$. The following result determines the $\gamma_{\mathrm{pr}}^{+}$-stability of a cycle of large order.

Theorem 5.4. For $n \geq 12$ and $n \notin\{13,14,17\}$,

$$
\text { st }_{\gamma_{\mathrm{pr}}}^{+}\left(C_{n}\right)= \begin{cases}2 & \text { when } n \equiv 0(\bmod 4) \\ 3 & \text { when } n(\bmod 4) \in\{2,3\} \\ 4 & \text { when } n \equiv 1(\bmod 4)\end{cases}
$$

As a consequence of Theorems 5.1 and 5.4, the paired domination stability of a cycle is determined.

Corollary 5.5. For $n \geq 3$,

$$
\operatorname{st}_{\gamma_{\mathrm{pr}}}\left(C_{n}\right)= \begin{cases}1 & \text { when } n \equiv 1(\bmod 4) \\ 2 & \text { when } n(\bmod 4) \in\{0,2\} \text { and } n \notin\{4,8\} \\ 3 & \text { when } n \equiv 3(\bmod 4) \\ 4 & \text { when } n \in\{4,8\}\end{cases}
$$

\section{Trees}

In this section, we first determine the $\gamma_{\mathrm{pr}}$-stability of trees in the family $\mathcal{F}_{\Delta}$ and a new family $\mathcal{E}_{\Delta}$.

Lemma 6.1. For $\Delta \geq 2$, if $T \in \mathcal{F}_{\Delta}$, then $\operatorname{st}_{\gamma_{\mathrm{pr}}}^{-}(T)=2 \Delta$. 
Proof of Lemma 6.1. Let $T$ be an arbitrary tree in the family $\mathcal{F}_{k, \Delta}$ for some $k \geq 2$ and $\Delta \geq 2$. We show that $\mathrm{st}_{\gamma_{\mathrm{pr}}}^{-}(T)=2 \Delta$. The family $\mathcal{F}_{k, 2}$ consists of all paths $P_{4 k}$ where $k \geq 2$. Therefore by Theorem 5.1, we have $\operatorname{st}_{\gamma_{\mathrm{pr}}}^{-}(T)=4=2 \Delta$ for each $T \in \mathcal{F}_{k, 2}$, which yields the desired result. Hence, we may assume that $\Delta \geq 3$. We show, by induction on $k \geq 2$, that every tree $T$ in the family $\mathcal{F}_{k, \Delta}$ satisfies st ${ }_{\gamma_{\mathrm{pr}}}^{-}(T)=2 \Delta$.

Suppose $k=2$, and so $T \in \mathcal{F}_{2, \Delta}$ (where recall that $\Delta \geq 3$ ). The tree $T$ can therefore be constructed from two vertex disjoint double stars $T_{1}$ and $T_{2}$, where $T_{i} \cong S(\Delta-1, \Delta-1)$ for $i \in[2]$, by selecting leaves $w_{1}$ and $w_{2}$ of $T_{1}$ and $T_{2}$, respectively, and adding the edge $w_{1} w_{2}$ to $T_{1} \cup T_{2}$. Let $x_{i}$ and $y_{i}$ be the two vertices of $T_{i}$ that are not leaves, where $x_{i} w_{i}$ is an edge. We note that $y_{1} x_{1} w_{1} w_{2} x_{2} y_{2}$ is a path in $T$. We note that $\gamma_{\mathrm{pr}}(T)=4$ and the set $\left\{x_{1}, x_{2}, y_{1}, y_{2}\right\}$ is a $\gamma_{\mathrm{pr}}$-set of $T$.

Let $S$ be a st $\bar{\gamma}_{\mathrm{pr}}-$ set of $G$. Thus, $S$ is a set in $\mathrm{NI}(G)$ with $|S|=\operatorname{st}_{\gamma_{\mathrm{pr}}}^{-}(G)$ such that $\gamma_{\mathrm{pr}}(T-S)=2$. Let $R$ be a $\gamma_{\mathrm{pr}}$-set of $T-S$, and so $R$ is a minimum PD-set of $T-S$ (of cardinality 2). Since $T[R]=P_{2}$, we note that $T-S$ is a tree of diameter at most 3. This implies that at most one of $x_{i}$ and $y_{3-i}$ belong to $T-S$ for $i \in[2]$. Thus, $\left|S \cap\left\{x_{i}, y_{3-i}\right\}\right| \geq 1$ for $i \in[2]$.

Suppose that $y_{1} \in S$ and $x_{2} \in S$. If $x_{1} \in S$, then all leaf neighbors of $y_{1}, x_{1}$ and $x_{2}$ belong to $S$, while if $y_{2} \in S$, then all leaf neighbors of $y_{1}, y_{2}$ and $x_{2}$ belong to $S$. In both cases, $|S| \geq 3 \Delta-2>2 \Delta$.

Suppose that $y_{1} \in S$ and $x_{2} \notin S$. If $y_{2} \in S$, then all leaf neighbors of $y_{1}$ and $y_{2}$ belong to $S$, implying that $|S| \geq 2 \Delta$. If $y_{2} \notin S$, then $x_{1} \in S$, implying that $S$ contains all leaf-neighbors of $y_{1}$ and $x_{1}$, and so $|S| \geq 2 \Delta-1$. However if in this case $|S|=2 \Delta-1$, implying that $\operatorname{diam}(T-S) \geq 4$, a contradiction. Hence, $|S| \geq 2 \Delta$.

Suppose that $y_{1} \notin S$ and $x_{2} \in S$. Since $T-S$ is a tree, $y_{2} \in S$ and all leaf neighbors of $y_{2}$ and $x_{2}$ belong to $S$, implying that $|S| \geq 2 \Delta-1$. However if in this case $|S|=$ $2 \Delta-1$, then $S$ contains $x_{2}$ and all leaf neighbors of $y_{1}$, implying that $\operatorname{diam}(T-S) \geq 4$, a contradiction. Hence, $|S| \geq 2 \Delta$. Therefore, in all three cases we have $|S| \geq 2 \Delta$, as desired. This proves the base case when $k=2$.

For the inductive hypothesis, let $k \geq 3$ and assume that if $T^{\prime} \in \mathcal{F}_{k^{\prime}, \Delta}$ where $2 \leq k^{\prime}<$ $k$, then $\mathrm{st}_{\gamma_{\mathrm{pr}}}^{-}\left(T^{\prime}\right)=2 \Delta$. We now consider a tree $T$ in the family $\mathcal{F}_{k, \Delta}$. Therefore, the tree $T$ can be constructed from $k$ vertex disjoint double stars $H_{1}, \ldots, H_{k}$, where $H_{i} \cong$ $S(\Delta-1, \Delta-1)$ for $i \in[k]$, by selecting one leaf $y_{i}$ from each double star $H_{i}$ and adding $k-1$ edges between vertices in $\left\{y_{1}, \ldots, y_{k}\right\}$ in such a way that the resulting graph is a tree with maximum degree $\Delta$. Let $w_{i}$ and $x_{i}$ be the two (adjacent) vertices of $H_{i}$ that are not leaves for $i \in[k]$, where $y_{i}$ is a leaf neighbor of $x_{i}$ for $i \in[k]$. We note that $\gamma_{\mathrm{pr}}(T)=2 k$ and the set $\cup_{i=1}^{k}\left\{w_{i}, x_{i}\right\}$ is the unique $\gamma_{\mathrm{pr}}$-set of $T$.

Let $U$ be the graph of order $k$ whose vertices correspond to the $k$ double stars $H_{1}, \ldots, H_{k}$ where two vertices are adjacent in $U$ if and only if the corresponding double stars are joined by an edge in $T$. We call $U$ the underlying graph of $T$. By construction, the graph $U$ is a tree, noting that $T$ is a tree. Let $V(U)=\left\{u_{1}, \ldots, u_{k}\right\}$ where $u_{i}$ is the vertex of $U$ corresponding to the double star $H_{i}$ for $i \in[k]$. Renaming the double stars if necessary, we may assume that $u_{1}$ is a leaf in $U$, and that $H_{1}$ is joined to $H_{2}$ in $T$. Thus, $y_{1} y_{2} \in E(T)$ and $y_{1} y_{j} \notin E(T)$ for $j \in[k] \backslash[2]$. We note that $w_{1} x_{1} y_{1} y_{2} x_{2} w_{2}$ is a path in $T$. Let $T^{\prime}=T-V\left(H_{1}\right)$. By construction, the tree $T^{\prime}$ belongs to the family $\mathcal{F}_{k^{\prime}, \Delta}$ where $k^{\prime}=k-1 \geq 2$. By induction, we have $\operatorname{st}_{\gamma_{\mathrm{pr}}}^{-}\left(T^{\prime}\right)=2 \Delta$.

Let $S$ be a st $\bar{\gamma}_{\mathrm{pr}}^{-}$-set of $T$. Thus, $S$ is a set in $\mathrm{NI}(T)$ with $|S|=\operatorname{st}_{\gamma_{\mathrm{pr}}}^{-}(T)$ such that $\gamma_{\mathrm{pr}}(T-S) \leq \gamma_{\mathrm{pr}}(T)-2=2 k-2$. Let $Q$ be a $\gamma_{\mathrm{pr}}$-set of $T-S$, and so $|Q| \leq 2 k-2$. 
Let $Q^{\prime}=Q \cap V\left(T^{\prime}\right)$ and $S^{\prime}=S \cap V\left(T^{\prime}\right)$. For $i \in[k]$, let $Q_{i}=Q \cap V\left(H_{i}\right)$ and $S_{i}=S \cap V\left(H_{i}\right)$. We proceed further with the following claim.

Claim 6.2. $|S| \geq 2 \Delta$.

Proof of Claim 6.2. Suppose, to the contrary, that $|S| \leq 2 \Delta-1$.

Subclaim 6.2.1. $\left|Q_{1}\right| \geq 2$.

Proof of Subclaim 6.2.1. Suppose, to the contrary, that $\left|Q_{1}\right| \leq 1$. Suppose that $Q_{1}=\emptyset$. In this case, $V\left(H_{1}\right) \backslash\left\{y_{1}\right\} \subseteq S_{1}$. If $y_{1} \in S_{1}$, then $\left|S_{1}\right|=2 \Delta>|S|$, a contradiction. Hence, $y_{1} \notin S_{1}$, and so $2 \Delta-1 \geq|S| \geq\left|S_{1}\right|=2 \Delta-1$, implying that $S=S_{1}$ and $|S|=2 \Delta-1$. In this case, a $\gamma_{\mathrm{pr}}$-set of $T-S$ contains at least one of $y_{1}$ and $y_{2}$. Since the set $\cup_{i=2}^{k}\left\{w_{i}, x_{i}\right\}$ is the unique $\gamma_{\mathrm{pr}}$-set of $T^{\prime}$, a $\gamma_{\mathrm{pr}}$-set of $T-S$ is therefore not a $\gamma_{\mathrm{pr}}$-set of $T^{\prime}$, and so $\gamma_{\mathrm{pr}}(T-S) \geq \gamma_{\mathrm{pr}}\left(T^{\prime}\right)+2=2(k-1)+2=2 k$, a contradiction. Hence, $\left|Q_{1}\right| \geq 1$.

By supposition, $\left|Q_{1}\right| \leq 1$. Consequently, $\left|Q_{1}\right|=1$, implying that $Q_{1}=\left\{y_{1}\right\}$ and $V\left(H_{1}\right) \backslash\left\{x_{1}, y_{1}\right\} \subseteq S_{1}$, and so $\left|S_{1}\right| \geq 2 \Delta-2$. If $x_{1} \in S_{1}$, then $\left|S_{1}\right|=2 \Delta-1$ and we end up in the previous case, which leads to a contradiction. Hence, $x_{1} \notin S_{1}$ and $x_{1} \notin Q_{1}$, implying that $y_{2} \in Q$ with the vertices $y_{1}$ and $y_{2}$ paired in $Q$, and $\left|S_{1}\right|=2 \Delta-2$. By supposition, $|S| \leq 2 \Delta-1$. If $|S|=2 \Delta-2$, then $S=S_{1}$ and $\gamma_{\mathrm{pr}}(T-S) \geq$ $\gamma_{\mathrm{pr}}\left(T^{\prime}\right)+2=2 k$, a contradiction. Hence, $|S|=2 \Delta-1$, and so the set $S$ contains a vertex $v^{\prime} \in V\left(T^{\prime}\right) \backslash\left\{y_{2}\right\}$. However noting that $\Delta \geq 3$, every non-isolating set of vertices of $T^{\prime}-y_{2}$ that decreases the paired domination number cannot contain only one vertex, implying that $\gamma_{\mathrm{pr}}(T-S) \geq\left|\left\{y_{1}, y_{2}\right\}\right|+\gamma_{\mathrm{pr}}\left(T^{\prime}-y_{2}\right)=2+\gamma_{\mathrm{pr}}\left(T^{\prime}\right)=2 k$, a contradiction.

Subclaim 6.2.2. $\left\{x_{1}, y_{1}\right\} \subseteq Q$.

Proof of Subclaim 6.2.2. Suppose, to the contrary, that $y_{1} \notin Q_{1}$, implying that $S^{\prime} \in$ $\mathrm{NI}\left(T^{\prime}\right)$. Recall that $S$ is a st $\overline{\mathrm{p}}_{\mathrm{pr}}$-set of $T$ and $\left|S^{\prime}\right| \leq|S| \leq 2 \Delta-1$. However, $\operatorname{st}_{\gamma_{\mathrm{pr}}}^{-}\left(T^{\prime}\right)=$ $2 \Delta$. Therefore, $\gamma_{\mathrm{pr}}\left(T^{\prime}-S^{\prime}\right) \geq \gamma_{\mathrm{pr}}\left(T^{\prime}\right)=2(k-1)$. Hence, $\gamma_{\mathrm{pr}}(T-S)=\gamma_{\mathrm{pr}}\left(T^{\prime}-S^{\prime}\right)+$ $\left|Q_{1}\right| \geq 2(k-1)+2=2 k$, a contradiction. Hence, $y_{1} \in Q_{1}$.

Suppose, to the contrary, that $x_{1} \notin Q_{1}$. Thus, all $\Delta-2$ leaf-neighbors of $x_{1}$ belong to the set $S_{1}$. By Claim 6.2.1, we have $\left|Q_{1}\right| \geq 2$. Hence, the set $Q_{1}$ contains $w_{1}$ and one of its leaf-neighbor $w_{1}^{\prime}$. We now consider the set $S^{*}=S \backslash S_{1}$. Since $S^{*} \in \mathrm{NI}(T)$ and $\left(Q \backslash\left\{w_{1}^{\prime}\right\}\right) \cup\left\{x_{1}\right\}$ is a PD-set of $T-S^{*}$, we have $\gamma_{\mathrm{pr}}\left(T-S^{*}\right) \leq|Q|=\gamma_{\mathrm{pr}}(T-S)$, contradicting our choice of the set $S$. Hence, $x_{1} \in Q_{1}$.

Subclaim 6.2.3. $w_{1} \notin Q_{1}$.

Proof of Subclaim 6.2.3. Suppose, to the contrary, that $w_{1} \in Q_{1}$. Hence, $\left\{w_{1}, x_{1}, y_{1}\right\} \subseteq$ $Q_{1}$, and so $S \cap V\left(H_{1}\right)=\emptyset$ by the minimality of $S$. Thus, $S=S^{\prime}$ and therefore $\left|S^{\prime}\right| \leq 2 \Delta-1$.

We show firstly that $x_{1}$ and $y_{1}$ are paired in $Q$. Suppose, to the contrary, that $x_{1}$ and $y_{1}$ are not paired in $Q$. This implies that $y_{2} \in Q$, and that $y_{1}$ and $y_{2}$ are paired in $Q$. Suppose that $x_{2} \notin S$, implying that $S^{\prime} \in \mathrm{NI}\left(T^{\prime}\right)$. By the minimality of the set $Q$, we have $x_{2} \notin Q$. Thus, the set $Q^{\prime} \cup\left\{x_{2}\right\}$ is a PD-set of $T^{\prime}-S^{\prime}$, and so $\left|Q^{\prime}\right|+1=\left|Q^{\prime} \cup\left\{x_{2}\right\}\right| \geq$ $\gamma_{\mathrm{pr}}\left(T^{\prime}-S^{\prime}\right) \geq \gamma_{\mathrm{pr}}\left(T^{\prime}\right)=2(k-1)$. Hence, $|Q|=\left|Q_{1}\right|+\left|Q^{\prime}\right| \geq 3+(2 k-3)=2 k=$ $\gamma_{\mathrm{pr}}(T)$, a contradiction. Hence, $x_{2} \in S$. We now consider the set $S^{*}=S \backslash\left\{x_{2}\right\}$. We note that $S^{*}$ is a non-isolating set of vertices of $T$, and the set $Q$ is a PD-set of $T-S^{*}$. Thus, 
$\gamma_{\mathrm{pr}}\left(T-S^{*}\right) \leq|Q| \leq 2 k-2$, which contradicts our choice of the set $S$. Hence, $x_{1}$ and $y_{1}$ are paired in $Q$.

Since $x_{1}$ and $y_{1}$ are paired in $Q$, the vertex $w_{1}$ is paired with one of its leaf neighbors, say $w_{1}^{\prime}$. By the minimality of $Q$ we note that $Q_{1}=\left\{w_{1}, w_{1}^{\prime}, x_{1}, y_{1}\right\}$. If $x_{2} \in Q$, then the set $Q \backslash\left\{w_{1}^{\prime}, y_{1}\right\}$ is a PD-set of $T-S$ (with $w_{1}$ and $x_{1}$ paired), contradicting the minimality of $Q$. Hence, $x_{2} \notin Q$. This in turn implies that $y_{2} \notin Q$. If $y_{2} \in S$, then once again we contradict the minimality of $Q$. Therefore, $y_{2} \notin S$. We remark, though, that possibly $x_{2} \in S$. Recall that by our earlier observations, $S=S^{\prime}$.

Let $S^{\prime \prime}=S \backslash\left\{x_{2}\right\}$. Thus, if $x_{2} \notin S$, then $S^{\prime \prime}=S$, while if $x_{2} \in S$, then $S^{\prime \prime}=S \backslash\left\{x_{2}\right\}$. The set $S^{\prime \prime}$ is a non-isolating set of $T^{\prime}$ such that $\left|S^{\prime \prime}\right| \leq|S| \leq 2 \Delta-1$. As observed earlier, $y_{2} \notin Q^{\prime}$ and $x_{2} \notin Q^{\prime}$. The set $Q^{\prime} \cup\left\{y_{2}, x_{2}\right\}$ is a PD-set of $T^{\prime}-S^{\prime \prime}$, implying that $\left|Q^{\prime}\right|+2 \geq \gamma_{\mathrm{pr}}\left(T^{\prime}-S^{\prime \prime}\right) \geq \gamma_{\mathrm{pr}}\left(T^{\prime}\right)=2(k-1)$. Hence, $\left|Q^{\prime}\right| \geq 2 k-4$, and so $|Q|=\left|Q_{1}\right|+\left|Q^{\prime}\right| \geq 4+(2 k-4)=2 k$, contradicting the fact that $|Q| \leq 2 k-2$.

Proof of Claim 6.2, continued: By Claim 6.2.3, $w_{1} \notin Q_{1}$. This implies that $Q_{1}=\left\{x_{1}, y_{1}\right\}$. The set $S_{1}$ therefore consists of the $\Delta-1$ leaf neighbors of $w_{1}$, and so $\left|S_{1}\right|=\Delta-1$. This is true for every leaf in the tree $U$. Hence, if $u_{i}$ is a leaf in $U$ for some $i \in[k]$, then in the corresponding double star $H_{i}$ of $T$ we have $Q_{i}=\left\{x_{i}, y_{i}\right\}$ and $\left|S_{i}\right|=\Delta-1$. Further, the set $S_{i}$ consists of the $\Delta-1$ leaf neighbors of $w_{i}$. In particular, $\left|Q_{1}\right|=2$ and $\left|S_{1}\right|=\Delta-1$. Since the underlying tree $U$ of $T$ has order $k \geq 3$, there are at least two leaves in $U$. Thus, $u_{p}$ is a leaf in $U$ for some $p \in[k] \backslash\{1\}$, implying that $\left|Q_{p}\right|=2$ and $\left|S_{p}\right|=\Delta-1$.

If $\left|Q_{i}\right| \geq 2$ for all $i \in[k]$, then $|Q| \geq 2 k$, a contradiction. Hence, $\left|Q_{q}\right| \leq 1$ for some $q \in[k]$. By our earlier observations, $u_{q}$ is not a leaf in the tree $U$, and so $q \notin\{1, p\}$. If $\left|Q_{q}\right|=0$, then $\left\{w_{q}, x_{q}\right\} \subseteq S_{q}$, and so $\left|S_{q}\right| \geq 2$ (in fact, $\left|S_{q}\right| \geq 2 \Delta-1$ ) and $|S| \geq\left|S_{1}\right|+\left|S_{p}\right|+\left|S_{q}\right| \geq(\Delta-1)+(\Delta-1)+2=2 \Delta$, a contradiction. Hence, $\left|Q_{q}\right|=1$, implying that $Q_{q}=\left\{y_{q}\right\}$ and $w_{q} \in S_{q}$, and so $\left|S_{q}\right| \geq 1$. Since the paired dominating number is an even integer and $|Q| \leq 2 k$, there exists $r \in[k] \backslash\{1, p, q\}$ such that $\left|Q_{r}\right|=1$. Therefore, $Q_{r}=\left\{y_{r}\right\}$ and $\left|S_{r}\right| \geq 1$. Hence, $|S| \geq\left|S_{1}\right|+\left|S_{p}\right|+\left|S_{q}\right|+\left|S_{r}\right| \geq$ $(\Delta-1)+(\Delta-1)+1+1=2 \Delta$, a contradiction. This completes the proof of Claim 6.2.

Proof of Lemma 6.1, continued: By Claim 6.2, we have $|S| \geq 2 \Delta$. By our choice of the set $S$, this implies that $\mathrm{st}_{\gamma_{\mathrm{pr}}}^{-}(T)=|S| \geq 2 \Delta$. Conversely, if we consider the set $S=V\left(H_{1}\right)$, then $S \in \mathrm{NI}(T)$ satisfies $|S|=2 \Delta$ and $\gamma_{\mathrm{pr}}(T-S)=\gamma_{\mathrm{pr}}\left(T^{\prime}\right)=2 k-2<\gamma_{\mathrm{pr}}(T)$, and so $\operatorname{st}_{\gamma_{\mathrm{pr}}}^{-}(T) \leq 2 \Delta$. Consequently, st $_{\gamma_{\mathrm{pr}}}^{-}(T)=2 \Delta$. This completes the proof of Lemma 6.1.

We determine next the $\gamma_{\mathrm{pr}}^{+}$-stability of a tree in the family $\mathcal{F}_{\Delta}$.

Lemma 6.3. For $\Delta \geq 2$, if $T \in \mathcal{F}_{\Delta}$, then $\operatorname{st}_{\gamma_{\mathrm{pr}}}^{+}(T) \leq \Delta-1$.

Proof. Let $T$ be an arbitrary tree in the family $\mathcal{F}_{k, \Delta}$ for some $k \geq 2$ and $\Delta \geq 2$. We use the same notation as in the proof of Lemma 6.1. In particular, $\gamma_{\mathrm{pr}}(T)=2 k$ and $H_{1}$ corresponds to a leaf $u_{1}$ in the underlying tree $U$ of $T$. Moreover, $y_{1} y_{2}$ is the edge joining $H_{1}$ and $H_{2}$ in $T$. Also, $w_{i}$ and $x_{i}$ are the support vertices in the double star $H_{i}$ and $w_{i} x_{i} y_{i}$ is a path in $H_{i}$ for $i \in[k]$. Let $L$ be the set of $\Delta-2$ leaf neighbors of $x_{1}$ in $T$, and let $S=L \cup\left\{x_{1}\right\}$. We resulting set $S \in N I(T)$ and the forest $T-S$ has two components, say $F_{1}$ and $F_{2}$ where $w_{1} \in V\left(F_{1}\right)$ and $y_{1} \in V\left(F_{2}\right)$. Moreover, $\gamma_{\mathrm{pr}}(T-S)=$ $\gamma_{\mathrm{pr}}\left(F_{1}\right)+\gamma_{\mathrm{pr}}\left(F_{2}\right)=2+2 k>\gamma_{\mathrm{pr}}(T)$. Therefore, st $_{\gamma_{\mathrm{pr}}}^{+}(T) \leq|S|=\Delta-1$. 
Recall that by Proposition 3.1, for $\Delta \geq 3$, if $T \in \mathcal{H}_{\Delta}$, then $\operatorname{st}_{\gamma_{\mathrm{pr}}}(T)=2 \Delta-1$. Further we remark that $\mathrm{st}_{\gamma_{\mathrm{pr}}}^{+}(T)=\infty$. We next define another family of trees $T$ with maximum degree $\Delta$ such that $\operatorname{st}_{\gamma_{\mathrm{pr}}}^{-}(T)=2 \Delta-1$. For integers $\Delta \geq 3$ and $\Delta-1 \geq k \geq 3$, let $E_{k, \Delta}$ be a graph obtained from the path $P_{2}$ with vertices $u$ and $v$ and the disjoint union of $2 k$ double stars $S(\Delta-1, \Delta-1)$ by selecting one leaf from each double star and identifying half of the selected leaves with the vertex $v$ and the other half of the selected leaves with the vertex $u$ (see Figure 4). Let

$$
\mathcal{E}_{\Delta}=\bigcup_{k \geq 3} E_{k, \Delta} .
$$

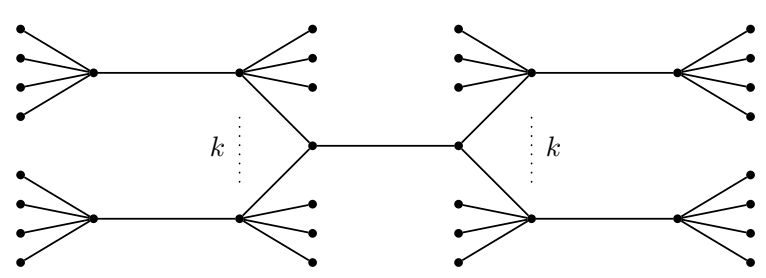

Figure 4: A tree $E_{k, 5}$ from the family $\mathcal{E}_{5}$.

If $T$ is a tree from the family $\mathcal{E}_{\Delta}$, then $\mathrm{st}_{\gamma_{\mathrm{pr}}}(T)=2 \Delta-1$. Moreover, if $T$ is isomorphic to the graph $E_{k, \Delta}$, then $\operatorname{st}_{\gamma_{\mathrm{pr}}}^{+}(T)=k(\Delta-1)$. In contrast to the family $\mathcal{H}_{\Delta}$, the trees from the family $\mathcal{E}_{\Delta}$ have finite $\gamma_{\mathrm{pr}}^{+}$-stability.

\section{Proof of Theorem 2.2}

In this section we present a proof of Theorem 2.2, which we restate below.

Theorem 2.2. If $T$ is a tree with maximum degree $\Delta$ satisfying $\gamma_{\mathrm{pr}}(T) \geq 4$, then the following hold.

(a) $\operatorname{st}_{\gamma_{\mathrm{pr}}}^{-}(T) \leq 2 \Delta$, with equality if and only if $T \in \mathcal{F}_{\Delta}$.

(b) st $_{\gamma_{\mathrm{pr}}}(T) \leq 2 \Delta-1$, and this bound is sharp for all $\Delta \geq 2$.

Proof. We first prove the statement given in part (a). Since $\gamma_{\mathrm{pr}}(T) \geq 4$, we have $\Delta \geq 2$. If $\Delta=2$, then $G$ is a path $P_{n}$ of order $n \geq 5$. In this case, the family $\mathcal{F}_{k, \Delta}=\left\{P_{n}: n \equiv\right.$ $0(\bmod 4)$ and $n \geq 8\}$, and Theorem 5.1 and Lemma 6.1 imply the desired result. Suppose, therefore, that $\Delta \geq 3$. The sufficiency of part (a) follows from Lemma 6.1. To prove the necessity, let $T$ be a tree with maximum degree $\Delta \geq 3$ satisfying $\gamma_{\mathrm{pr}}(T) \geq 4$. Let $d=\operatorname{diam}(T)$, and so $d \geq 4$. Let $P: v_{0} v_{1} \ldots v_{d}$ be a diametral path in $G$. Thus, $v_{0}$ and $v_{d}$ are leaves in $T$ and $d\left(v_{0}, v_{d}\right)=\operatorname{diam}(G)$. We now consider the tree $T$ rooted at the vertex $v_{d}$. Let $D$ be a $\gamma_{\mathrm{pr}}$-set of $T$.

Suppose that there is a child $u_{1}$ of $v_{2}$ that is a support vertex in $T$ where $u_{1} \neq v_{1}$. Let $u_{0}$ be a leaf neighbor of $u_{1}$. Since every PD-set of $T$ contains all support vertices, we have $\left\{v_{1}, u_{1}\right\} \subset D$. Renaming vertices if necessary, we may assume that $u_{0}$ and $u_{1}$ are paired in $D$. Thus, if $S$ consists of the vertex $u_{1}$ and all leaf neighbors of $u_{1}$, then $S \in \operatorname{NI}(T)$ and $\gamma_{\mathrm{pr}}(T-S) \leq|D|-2=\gamma_{\mathrm{pr}}(T)-2$. Hence, $\mathrm{st}_{\gamma_{\mathrm{pr}}}^{-}(T) \leq|S| \leq \Delta<2 \Delta-1$, and the desired result follows. Assume, therefore, that every child of $v_{2}$ different from $v_{1}$ is a leaf. 
Suppose that there is a $\gamma_{\mathrm{pr}}$-set, $D_{2,3}$, of $T$ such that $v_{2}$ and $v_{3}$ are paired in $D_{2,3}$. Necessarily, $v_{1} \in D_{2,3}$ and $v_{1}$ is paired in $D_{2,3}$ with one of its leaf neighbors. Let $S$ consist of the vertex $v_{1}$ and all of its leaf neighbors. Thus, $S \in \mathrm{NI}(T)$ and $\gamma_{\mathrm{pr}}(T-S) \leq$ $\left|D_{2,3}\right|-2=\gamma_{\mathrm{pr}}(T)-2<\gamma_{\mathrm{pr}}(T)$, implying that $\mathrm{st}_{\gamma_{\mathrm{pr}}}^{-}(T) \leq|S| \leq \Delta<2 \Delta-1$, once again implying the desired result. Therefore, we may assume that in every $\gamma_{\mathrm{pr}}$-set of $T$ the vertices $v_{2}$ and $v_{3}$ are not paired.

Suppose that there is a $\gamma_{\mathrm{pr}}$-set, $D_{3}$, of $T$ which contains a neighbor of $v_{3}$ different from $v_{2}$. In this case, if $S$ consists of the vertex $v_{2}$ and all its descendants, then $|S| \leq 2 \Delta-1, S \in \mathrm{NI}(T)$ and $\gamma_{\mathrm{pr}}(T-S) \leq\left|D_{3}\right|-2=\gamma_{\mathrm{pr}}(T)-2<\gamma_{\mathrm{pr}}(T)$, noting that the set $D_{3} \backslash S$ is a PD-set of $T-S$ and, by the minimality of $D_{3}$ we have $\left|D_{3} \cap S\right|=2$. Thus, $\operatorname{st}_{\gamma_{\mathrm{pr}}}^{-}(T) \leq|S| \leq 2 \Delta-1$, and the desired result follows. Hence, we may assume that every $\gamma_{\mathrm{pr}}$-set of $T$ contains the vertex $v_{2}$ but no other vertex in $N\left[v_{3}\right]$. In particular, $N\left[v_{3}\right] \cap D=\left\{v_{2}\right\}$.

Suppose that $d_{T}\left(v_{1}\right)<\Delta$ or $d_{T}\left(v_{2}\right)<\Delta$. Thus, $d_{T}\left(v_{1}\right)+d_{T}\left(v_{2}\right) \leq 2 \Delta-1$. In order to dominate the vertex $v_{0}$, we have $v_{1} \in D$. By our earlier assumptions, $v_{2} \in D$ and every child of $v_{2}$ different from $v_{1}$ is a leaf. Thus by the minimality of the set $D$, the vertex $v_{1}$ is the only descendant of $v_{2}$ that belongs to the set $D$, and the vertices $v_{1}$ and $v_{2}$ are paired in $D$. Hence, if $S=N\left[v_{2}\right] \cup N\left[v_{1}\right]$, then $S \in \mathrm{NI}(T)$ and $|S|=d_{T}\left(v_{1}\right)+d_{T}\left(v_{2}\right) \leq 2 \Delta-1$. Further, $D \backslash\left\{v_{1}, v_{2}\right\}$ is a PD-set of $T-S$, and so $\gamma_{\mathrm{pr}}(T-S) \leq|D|-2=\gamma_{\mathrm{pr}}(T)-2$, implying that $\operatorname{st}_{\gamma_{\mathrm{pr}}}^{-}(T) \leq|S| \leq 2 \Delta-1$, yielding the desired result. Hence, we may assume that $d_{T}\left(v_{1}\right)=d_{T}\left(v_{2}\right)=\Delta$.

Suppose that $d_{T}\left(v_{3}\right) \geq 3$, and let $u_{2}$ be a child of $v_{3}$ different from $v_{2}$. If $u_{2}$ is a leaf, then $v_{3}$ belongs to every $\gamma_{\mathrm{pr}}$-set of $T$, while if $u_{2}$ is not a leaf, then from the structure of the rooted tree $T$ the vertex $u_{2}$ can be chosen to belong to some $\gamma_{\mathrm{pr}}$-set of $T$. In both cases, we contradict our earlier assumption that every $\gamma_{\mathrm{pr}}$-set of $T$ contains the vertex $v_{2}$ but no other vertex in $N\left[v_{3}\right]$. Hence, $d_{T}\left(v_{3}\right)=2$. We now let $S=N\left[v_{1}\right] \cup N\left[v_{2}\right]$, and so $S \in \mathrm{NI}(T)$ and $|S|=d_{T}\left(v_{1}\right)+d_{T}\left(v_{2}\right)$. By our earlier observations, $|S|=2 \Delta$ and $\gamma_{\mathrm{pr}}(T-S)=\gamma_{\mathrm{pr}}(T)-2<\gamma_{\mathrm{pr}}(T)$, implying that $\mathrm{st}_{\gamma_{\mathrm{pr}}}^{-}(T) \leq|S|=2 \Delta$. This proves the desired upper bound.

We show next that if we have equality in the upper bound in part (a), then $T \in \mathcal{F}_{\Delta}$. Let $\operatorname{st}_{\gamma_{\mathrm{pr}}}^{-}(T)=2 \Delta$. By our earlier observations, we have that every child of $v_{2}$ different from $v_{1}$ is a leaf. Further, $d_{T}\left(v_{1}\right)=d_{T}\left(v_{2}\right)=\Delta$ and $d_{T}\left(v_{3}\right)=2$. We now re-root the tree $T$ at the vertex $v_{0}$, thereby interchanging the roles of $v_{0}$ and $v_{d}$. Identical arguments as before show that every child of $v_{d-2}$ different from $v_{d-1}$ is a leaf. Further, $d_{T}\left(v_{d-1}\right)=$ $d_{T}\left(v_{d-2}\right)=\Delta$ and $d_{T}\left(v_{d-3}\right)=2$. In particular, $d \geq 6$.

Suppose that $d=6$, and so $v_{d-3}=v_{3}$. In this case, the tree $T$ is determined and $\gamma_{\mathrm{pr}}(T)=4$. Letting $S=\left(N\left[v_{1}\right] \cup N\left[v_{2}\right]\right) \backslash\left\{v_{3}\right\}$, we have $S \in \mathrm{NI}(T)$ and $|S|=d_{T}\left(v_{1}\right)+d_{T}\left(v_{2}\right)-1=2 \Delta-1$. Further, $\gamma_{\mathrm{pr}}(T-S)=2<\gamma_{\mathrm{pr}}(T)$. Therefore, $\operatorname{st}_{\gamma_{\mathrm{pr}}}^{-}(T) \leq|S|=2 \Delta-1$, a contradiction. Hence, $d \geq 7$, and so $v_{d-3} \neq v_{3}$.

We now consider the tree $T^{\prime}=T-\left(N\left[v_{1}\right] \cup N\left[v_{2}\right]\right)$. If $\gamma_{\mathrm{pr}}\left(T^{\prime}\right)=2$, then by our earlier observations, we have $d=7$ and $T^{\prime} \cong S(\Delta-1, \Delta-1)$ where $v_{d-1}$ and $v_{d-2}$ are the two (adjacent) vertices in $T^{\prime}$ that are not leaves. Therefore, $T \in T_{2, \Delta}$, and so $T \in$ $T_{\Delta}$. Hence, we may assume that $\gamma_{\mathrm{pr}}\left(T^{\prime}\right) \geq 4$, for otherwise the desired characterization follows. In particular, $d \geq 8$. As observed earlier, $d_{T}\left(v_{d-1}\right)=d_{T}\left(v_{d-2}\right)=\Delta$, implying that $\Delta\left(T^{\prime}\right)=\Delta$ and $\operatorname{st}_{\gamma_{\mathrm{pr}}}^{-}\left(T^{\prime}\right) \leq 2 \Delta$.

Let $D$ be a $\gamma_{\mathrm{pr}}$-set of $T$. Since every PD-set of $T$ contains the set of support vertices, we note that $v_{1}, v_{2} \in D$. By the minimality of $D$, no leaf-neighbor of $v_{1}$ or $v_{2}$ belongs to 
$D$. If $v_{3} \in D$, then $v_{4} \in D$ (with $v_{3}$ and $v_{4}$ paired in $D$ ). However in this case, we can replace $v_{3}$ in $D$ with an arbitrary neighbor of $v_{4}$ that does not belong to $D$. Hence, we can choose the $\gamma_{\mathrm{pr}}$-set $D$ of $T$ so that $v_{3} \notin D$. The resulting set $D$ when restricted to $V\left(T^{\prime}\right)$ is a PD-set of $T^{\prime}$, implying that $\gamma_{\mathrm{pr}}\left(T^{\prime}\right) \leq|D|-2=\gamma_{\mathrm{pr}}(T)-2$. Conversely, every PD-set of $T^{\prime}$ can be extended to a PD-set of $T$ by adding to it the vertices $v_{1}$ and $v_{2}$ (with $v_{1}$ and $v_{2}$ paired), and so $\gamma_{\mathrm{pr}}(T) \leq \gamma_{\mathrm{pr}}\left(T^{\prime}\right)+2$. Consequently, $\gamma_{\mathrm{pr}}(T)=\gamma_{\mathrm{pr}}\left(T^{\prime}\right)+2$.

Suppose that $\mathrm{st}_{\gamma_{\mathrm{pr}}}^{-}\left(T^{\prime}\right)<2 \Delta$. Let $S^{\prime}$ be a st $\bar{\gamma}_{\mathrm{pr}}$-set of $T^{\prime}$. Thus, $S$ is a set in $\mathrm{NI}\left(T^{\prime}\right)$ with $\left|S^{\prime}\right|=\operatorname{st}_{\gamma_{\mathrm{pr}}}^{-}\left(T^{\prime}\right)<2 \Delta$ such that $\gamma_{\mathrm{pr}}\left(T-S^{\prime}\right)<\gamma_{\mathrm{pr}}\left(T^{\prime}\right)$. If $D^{\prime}$ is a $\gamma_{\mathrm{pr}}$-set of $T^{\prime}-S^{\prime}$, then $D^{\prime} \cup\left\{v_{1}, v_{2}\right\}$ is a PD-set of $T-S$, and so $\gamma_{\mathrm{pr}}\left(T-S^{\prime}\right) \leq\left|D^{\prime}\right|+2=\gamma_{\mathrm{pr}}\left(T-S^{\prime}\right)+2<$ $\gamma_{\mathrm{pr}}\left(T^{\prime}\right)+2=\gamma_{\mathrm{pr}}(T)$. Hence, $S^{\prime} \in \mathrm{NI}(T)$ and $\gamma_{\mathrm{pr}}\left(T-S^{\prime}\right)<\gamma_{\mathrm{pr}}\left(T^{\prime}\right)$, implying that st $_{\gamma_{\mathrm{pr}}}^{-}(T) \leq\left|S^{\prime}\right|=\mathrm{st}_{\gamma_{\mathrm{pr}}}^{-}\left(T^{\prime}\right)<2 \Delta$, a contradiction. Therefore, $\mathrm{st}_{\gamma_{\mathrm{pr}}}^{-}\left(T^{\prime}\right)=2 \Delta$.

Hence, the tree $T^{\prime}$ satisfies $\Delta\left(T^{\prime}\right)=\Delta, \gamma_{\mathrm{pr}}\left(T^{\prime}\right) \geq 4$ and $\mathrm{st}_{\gamma_{\mathrm{pr}}}^{-}\left(T^{\prime}\right)=2 \Delta$. Proceeding by induction, we have $T^{\prime} \in \mathcal{F}_{\Delta}$. Thus, $T^{\prime}$ is constructed from the disjoint union of $k^{\prime}$ double stars each isomorphic to $S(\Delta-1, \Delta-1)$, by selecting one leaf from each double star and adding $k^{\prime}-1$ edges between these selected leaves to produce a tree with maximum degree $\Delta$. The resulting tree $T^{\prime}$ satisfies $\gamma_{\mathrm{pr}}\left(T^{\prime}\right)=2 k^{\prime}$ with the $2 k^{\prime}$ support vertices forming a $\gamma_{\mathrm{pr}}$-set of $T^{\prime}$.

By construction of $T^{\prime}$, the tree $T^{\prime}$ contains the vertex $v_{4}$ but not the vertex $v_{3}$. Suppose that $v_{4}$ is a support vertex in $T^{\prime}$, implying by construction of $T^{\prime}$ that $v_{4}$ is a vertex of degree $\Delta$ in $T^{\prime}$. Let $S=\left(N\left[v_{1}\right] \cup N\left[v_{2}\right]\right) \backslash\left\{v_{3}\right\}$. We note that $S \in \mathrm{NI}(T)$ and $|S|=2 \Delta-1$. Let $D^{\prime}$ be the (unique) $\gamma_{\mathrm{pr}}$-set of $T^{\prime}$, and so $D^{\prime}$ is the set of $2 k^{\prime}$ support vertices in $T^{\prime}$. In particular, we note that $v_{4} \in D^{\prime}$. The set $D^{\prime}$ is a PD-set of $T-S$, and so $\gamma_{\mathrm{pr}}(T-S) \leq\left|D^{\prime}\right|=\gamma_{\mathrm{pr}}\left(T^{\prime}\right)=\gamma_{\mathrm{pr}}(T)-2$. Therefore, st $_{\gamma_{\mathrm{pr}}}^{-}(T) \leq|S|=2 \Delta-1$, a contradiction. Hence, $v_{4}$ is a leaf of $T^{\prime}$, and so $v_{4}$ is a leaf in one of the $k^{\prime}$ double stars in the construction of $T^{\prime}$. Selecting the leaf $v_{4}$ from this double star and selecting the leaf $v_{3}$ from the double star induced by $N\left[v_{1}\right] \cup N\left[v_{2}\right]$, which is isomorphic to $S(\Delta-1, \Delta-1)$, and adding back the edge $v_{3} v_{4}$ we re-construct the tree $T$, showing that $T \in \mathcal{F}_{\Delta}$. This completes the proof of part (a).

Part (b) now follows readily from part (a). If $T \in \mathcal{F}_{\Delta}$ for some $\Delta \geq 2$, then by Lemmas 6.1 and 6.3, we have $\operatorname{st}_{\gamma_{\mathrm{pr}}}(T) \leq \Delta-1$. Hence, we may assume that $T \notin \mathcal{F}_{\Delta}$ for any $\Delta \geq 2$, for otherwise the bound in part (b) is immediate. With this assumption, the upper bound in part (b) follows immediately from part (a) noting that st $\gamma_{\mathrm{pr}}(T) \leq \operatorname{st}_{\gamma_{\mathrm{pr}}}(T) \leq$ $2 \Delta-1$. That the bound is tight for all $\Delta \geq 2$ follows from Proposition 3.1.

\section{Proof of Theorem 2.3}

In this section we present a proof of Theorem 2.3, which we restate below.

Theorem 2.3. If $G$ is a connected graph with $\gamma_{\mathrm{pr}}(G) \geq 4$, then $\mathrm{st}_{\gamma_{\mathrm{pr}}}^{-}(G) \leq 2 \Delta(G)$, and this bound is sharp.

Proof. Let $G$ be a connected graph with $\gamma_{\mathrm{pr}}(G) \geq 4$ and let $\Delta=\Delta(G)$. Since $\gamma_{\mathrm{pr}}(G) \geq 4$, we have $\Delta \geq 2$. If $\Delta=2$, then $G$ is a path $P_{n}$ or a cycle $C_{n}$, and by Theorem 5.1, we have $\operatorname{st}_{\gamma_{\mathrm{pr}}}^{-}(G) \leq 2 \Delta$, with equality if and only if $n \equiv 0(\bmod 4)$. Assume, therefore, that $\Delta \geq 3$.

Let $T$ be a spanning tree of $G$ such that $\gamma_{\mathrm{pr}}(T)=\gamma_{\mathrm{pr}}(G)$. We note that such a tree exists by Lemma 4.1. Let $S$ be a st $\bar{\gamma}_{\mathrm{pr}}$-set of $T$. Thus, $S$ is a set in $\mathrm{NI}(T)$ with $|S|=\operatorname{st}_{\gamma_{\mathrm{pr}}}^{-}(T)$ such that $\gamma_{\mathrm{pr}}(T-S)<\gamma_{\mathrm{pr}}(T)$. By Observation 4.2, we have 
$|S|=$ st $_{\gamma_{\mathrm{pr}}}^{-}(T) \leq n-2$. Since $S \in \mathrm{NI}(T)$, every vertex in $T-S$, and therefore in the supergraph $G-S$, has degree at least 1 . Hence, $S \in \mathrm{NI}(G)$ and since $\gamma_{\mathrm{pr}}(G-S) \leq$ $\gamma_{\mathrm{pr}}(T-S)$, we have $\gamma_{\mathrm{pr}}(G-S)<\gamma_{\mathrm{pr}}(G)$. Thus, $\mathrm{st}_{\gamma_{\mathrm{pr}}}^{-}(G) \leq|S|=\mathrm{st}_{\gamma_{\mathrm{pr}}}^{-}(T)$. By Theorem 2.2, we have $\mathrm{st}_{\gamma_{\mathrm{pr}}}^{-}(T) \leq 2 \Delta(T)$. Noting that $\Delta(T) \leq \Delta(G)$, we therefore have that st $_{\gamma_{\mathrm{pr}}}^{-}(G) \leq \mathrm{st}_{\gamma_{\mathrm{pr}}}^{-}(T) \leq 2 \Delta(T) \leq 2 \Delta(G)=2 \Delta$.

To show that the upper bound in Theorem 2.3 is tight, we present a family of graphs with maximum degree $\Delta$ and $\gamma_{\mathrm{pr}}(G) \geq 4$ satisfying $\operatorname{st}_{\gamma_{\mathrm{pr}}}^{-}(G)=2 \Delta$. Our first family, $\mathcal{G}_{\Delta}$, is constructed as follows. For $k \geq 2$ and $\Delta \geq 2$, let $G_{k, \Delta}$ be a graph obtained from $k$ double stars $S(\Delta-1, \Delta-1)$ by choosing two leaves at distance 3 apart in each double star and adding $k$ edges between the chosen leaves in such a way, that every chosen vertex has degree 2 in the resulting graph. Let $\mathcal{G}_{\Delta}$ be the family of all such graphs $G_{k, \Delta}$ for all $k \geq 2$. The graph $G_{2,6} \in \mathcal{G}_{6}$, for example, is illustrated in Figure 5. We note that $\gamma_{\mathrm{pr}}\left(G_{k, \Delta}\right)=2 k$ and that set of $2 k$ vertices of degree $\Delta$ is the unique $\gamma_{\mathrm{pr}}$-set of $G_{k, \Delta}$. Furthermore, $\operatorname{st}_{\gamma_{\mathrm{pr}}}^{-}\left(G_{k, \Delta}\right)=2 \Delta$.

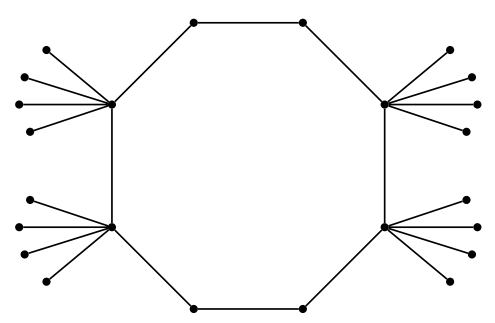

Figure 5: The graph $G_{2,6}$ from a class of graphs $G_{k, \Delta}$.

Recall that by definition we have $\operatorname{st}_{\gamma_{\mathrm{pr}}}(G) \leq \operatorname{st}_{\gamma_{\mathrm{pr}}}^{-}(G)$ for every graph $G$. Hence, as an immediate consequence of Theorem 2.3 we have Corollary 2.4. Recall its statement.

Corrolary 2.4. If $G$ is a connected graph with $\gamma_{\mathrm{pr}}(G) \geq 4$, then $\operatorname{st}_{\gamma_{\mathrm{pr}}}(G) \leq 2 \Delta(G)$.

It remains an open problem, however, to determine if the upper bound of Corollary 2.4 is best achievable for all values of possible value of $\Delta(G)=\Delta \geq 2$. If $\Delta=2$ and $G$ is a path, then $G \cong P_{n}$ where $n \geq 5$, and $\operatorname{st}_{\gamma_{\mathrm{pr}}}(G) \leq 2 \Delta-2$ by Corollary 5.3. If $\Delta=2$ and $G$ is a cycle, then $G \cong C_{n}$ where $n \geq 5$, and $\operatorname{st}_{\gamma_{\mathrm{pr}}}(G) \leq 2 \Delta$ by Corollary 5.5 , with equality if and only if $G=C_{8}$. Hence, the only connected graph $G$ with maximum degree $\Delta=2$ satisfying $\gamma_{\mathrm{pr}}(G) \geq 4$ and $\operatorname{st}_{\gamma_{\mathrm{pr}}}(G)=2 \Delta$ is the 8-cycle, namely $G=C_{8}$. For $\Delta \geq 3$, we do not know of a connected graph $G$ with maximum degree $\Delta$ satisfying $\gamma_{\mathrm{pr}}(G) \geq 4$ and $\operatorname{st}_{\gamma_{\mathrm{pr}}}(G)=2 \Delta$.

By Corollary 5.5 and Proposition 3.1, for any given $\Delta \geq 2$, there do exists infinite families of connected graphs $G$ with maximum degree $\Delta$ satisfying st $\gamma_{\mathrm{pr}}(G)=2 \Delta-1$. Thus, if the upper bound of Corollary 2.4 can be improved to $\operatorname{st}_{\gamma_{\mathrm{pr}}}(G) \leq 2 \Delta-1$ in the case when $\Delta \geq 3$, then this bound would be tight.

\section{ORCID iDs}


Monika Pilśniak (D) https://orcid.org/0000-0002-3734-7230

Elżbieta Tumidajewicz (D) https://orcid.org/0000-0002-1413-2413

\section{References}

[1] M. Amraee, N. Jafari Rad and M. Maghasedi, Roman domination stability in graphs, Math. Rep. (Bucur.) 21 (2019), 193-204, http://imar.ro/journals/Mathematical_ Reports/php/2019/Mrc19_2.php.

[2] A. Aytaç and B. Atay Atakul, Exponential domination critical and stability in some graphs, Int. J. Found. Comput. Sci. 30 (2019), 781-791, doi:10.1142/s0129054119500217.

[3] D. Bauer, F. Harary, J. Nieminen and C. L. Suffel, Domination alteration sets in graphs, Discrete Math. 47 (1983), 153-161, doi:10.1016/0012-365x(83)90085-7.

[4] R. C. Brigham, P. Z. Chinn and R. D. Dutton, Vertex domination-critical graphs, Networks 18 (1988), 173-179, doi:10.1002/net.3230180304.

[5] R. C. Brigham, T. W. Haynes, M. A. Henning and D. F. Rall, Bicritical domination, Discrete Math. 305 (2005), 18-32, doi:10.1016/j.disc.2005.09.013.

[6] T. Burton and D. P. Sumner, Domination dot-critical graphs, Discrete Math. 306 (2006), 11-18, doi:10.1016/j.disc.2005.06.029.

[7] W. J. Desormeaux, T. W. Haynes and M. A. Henning, Total domination changing and stable graphs upon vertex removal, Discrete Appl. Math. 159 (2011), 1548-1554, doi:10.1016/j.dam. 2011.06.006.

[8] W. J. Desormeaux, T. W. Haynes and M. A. Henning, Paired domination in graphs, in: T. W. Haynes, S. T. Hedetniemi and M. A. Henning (eds.), Topics in Domination in Graphs, Springer, Cham, volume 64 of Dev. Math., pp. 31-77, 2020, doi:10.1007/978-3-030-51117-3\_3.

[9] O. Favaron, D. P. Sumner and E. Wojcicka, The diameter of domination $k$-critical graphs, $J$. Graph Theory 18 (1994), 723-734, doi:10.1002/jgt.3190180708.

[10] T. W. Haynes, S. T. Hedetniemi and M. A. Henning (eds.), Topics in Domination in Graphs, volume 64 of Developments in Mathematics, Springer, Cham, 2020, doi:10.1007/ 978-3-030-51117-3.

[11] T. W. Haynes and P. J. Slater, Paired-domination in graphs, Networks 32 (1998), 199-206, doi:10.1002/(sici)1097-0037(199810)32:3<199::aid-net4>3.0.co;2-f.

[12] M. A. Henning and M. Krzywkowski, Total domination stability in graphs, Discrete Appl. Math. 236 (2018), 246-255, doi:10.1016/j.dam.2017.07.022.

[13] M. A. Henning and N. J. Rad, On total domination vertex critical graphs of high connectivity, Discrete Appl. Math. 157 (2009), 1969-1973, doi:10.1016/j.dam.2008.12.009.

[14] M. A. Henning and A. Yeo, Total Domination in Graphs, Springer Monographs in Mathematics, Springer, New York, 2013, doi:10.1007/978-1-4614-6525-6.

[15] N. Jafari Rad, E. Sharifi and M. Krzywkowski, Domination stability in graphs, Discrete Math. 339 (2016), 1909-1914, doi:10.1016/j.disc.2015.12.026.

[16] Z. Li, Z. Shao and S.-j. Xu, 2-rainbow domination stability of graphs, J. Comb. Optim. 38 (2019), 836-845, doi:10.1007/s10878-019-00414-0.

[17] D. P. Sumner, Critical concepts in domination, Discrete Math. 86 (1990), 33-46, doi:10.1016/ 0012-365x(90)90347-k.

[18] D. P. Sumner and P. Blitch, Domination critical graphs, J. Comb. Theory Ser. B 34 (1983), 65-76, doi:10.1016/0095-8956(83)90007-2. 